\title{
Suspended Sediment Loads and Tributary Inputs into the Mississippi River below St. Louis, MO, 1990-2013: A Comparison with the Keown et al. (1981) Report
}

MRG\&P Report No. 12 • July 2017

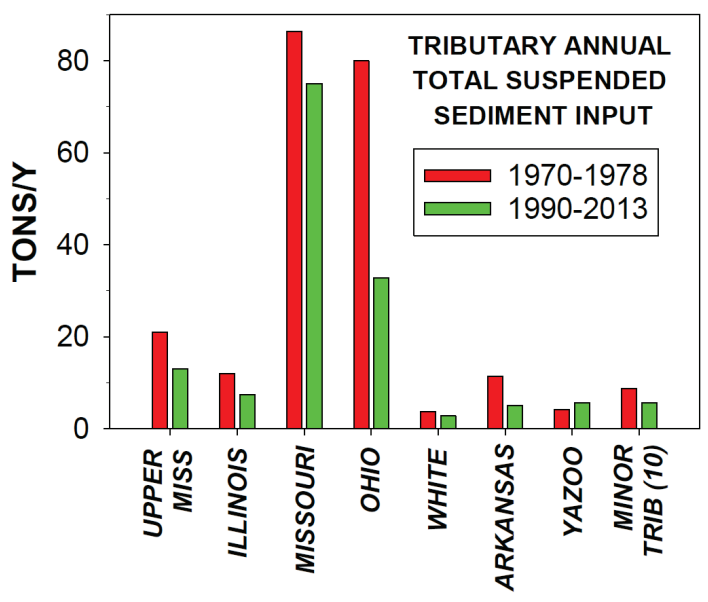

MRG\&P

Mississippi River Geomorphology \& Potamology Program

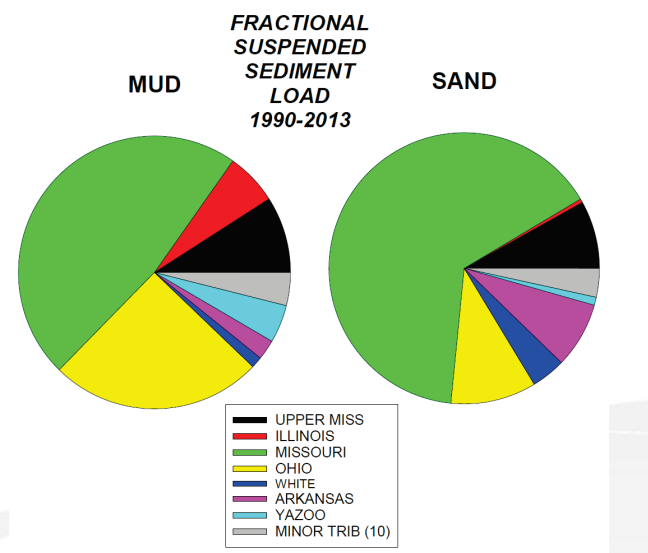




\title{
Suspended Sediment Loads and Tributary Inputs into the Mississippi River below St. Louis, MO, 1990-2013: A Comparison with the Keown et al. (1981) Report
}

\author{
Mead A. Allison \\ The Water Institute of the Gulf \\ 301 N. Main Street, Suite 2000 \\ Baton Rouge, LA 70825 \\ David S. Biedenharn \\ U.S. Army Engineer Research and Development Center \\ Coastal and Hydraulics Laboratory \\ 3909 Halls Ferry Road \\ Vicksburg, MS 39180-6199 \\ Charles D. Little, Jr. \\ Mendrop Resources Engineering, LLC \\ 854 Wilson Drive, Suite $A$ \\ Ridgeland, MS 39157
}

Final report

Approved for public release; distribution is unlimited.

\author{
Prepared for U.S. Army Corps of Engineers, Mississippi Valley Division \\ Mississippi River Geomorphology and Potamology Program \\ 1400 Walnut Street \\ Vicksburg, MS 39180 \\ Under Project 127672, "Geomorphic Assessments"
}




\section{Abstract}

Annual suspended sediment loads and water discharges were calculated in the Mississippi River main-stem channel and major tributary inputs at gaging stations from St. Louis, MO, to Belle Chasse, LA, for the period of water years 1990 to 2013. The purpose was to (1) quantify changes in the Mississippi River sediment budget by linking with an earlier study for the periods of pre-1953 and 1970-1978 (Keown et al. 1981) and (2) examine the role of anthropogenic (e.g., dams, river control works, soil conservation practices) and natural (e.g., rainfall and denudation rates) factors in controlling these changes. The present report focuses on (1) a first-order comparison of changes in station-specific sediment loads with the earlier Keown results, (2) documenting data quality and data comparison issues observed in the data, and (3) identifying possible additional gaging station sites at critical junctures that would improve future sediment budget assessments for the Mississippi River. It is anticipated that sediment budgets will provide a powerful framework when combined with other geomorphic assessment tools (e.g., specific gage records, channel geometry changes) to aid in unraveling the complex morphological processes that drive the Mississippi River. This integration is also planned for future, comprehensive MRG\&P efforts.

DISCLAIMER: The contents of this report are not to be used for advertising, publication, or promotional purposes. Citation of trade names does not constitute an official endorsement or approval of the use of such commercial products. All product names and trademarks cited are the property of their respective owners. The findings of this report are not to be construed as an official Department of the Army position unless so designated by other authorized documents. 


\section{Contents}

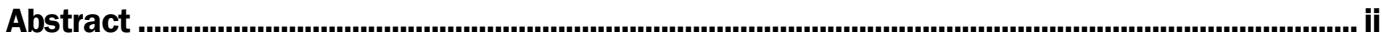

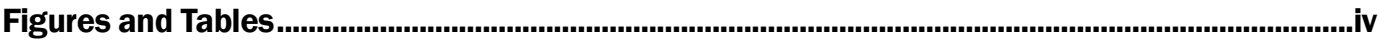

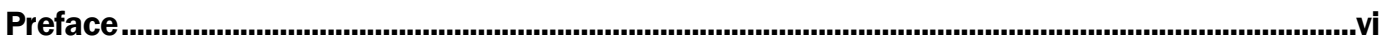

Unit Conversion Factors ................................................................................................................................vii

1 Background .................................................................................................................................... 1

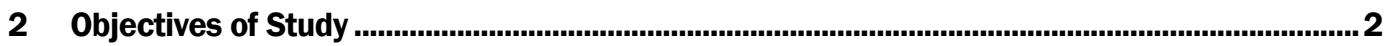

3 Methodology: Suspended Sediment Discharge Analysis ................................................... 5

3.1 Data sources............................................................................................... 5

3.2 Method for calculating water discharge ........................................................... 14

3.3 Sediment discharge calculations ................................................................... 15

3.4 Sediment discharge interpretation .............................................................. 19

4 Discussion of Results .............................................................................................................25

4.1 Water and suspended sediment loads for WY 1990-2013 for the Mississippi River and major tributaries ...................................................................... 25

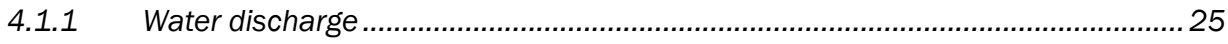

4.1.2 Total and sand-sized suspended sediment discharge.........................................29

4.2 A comparison of suspended sediment loads in WY 1990-2013 with WY

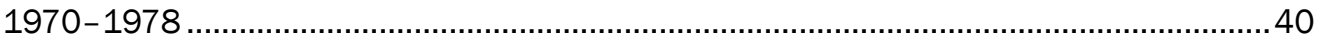

4.3 Future network design and station operation ................................................45

5 Summary .....................................................................................................................................51

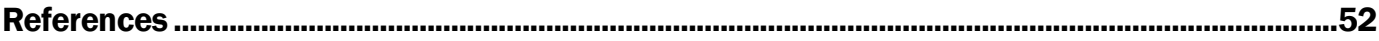

\section{Report Documentation Page}




\section{Figures and Tables}

\section{Figures}

Figure 1. Map of the location of Mississippi River stations from St. Louis, MO, to the Gulf of Mexico utilized in the present analysis.

Figure 2. Map of the location of Mississippi River tributary stations north of St. Louis, MO, utilized in the present analysis.

Figure 3. Map of the location of Mississippi River tributary stations south of St. Louis, MO, utilized in the present analysis.

Figure 4. Water ratings curve (stage-discharge) for the Mississippi River (USGS) station at Baton Rouge, LA.

Figure 5. Sediment ratings curve for the Mississippi River at Thebes, IL, (total and sand fraction) constructed using boat-based data on suspended sediment load from the USGS NWIS.

Figure 6. Annual average water discharge (in $10^{11} \mathrm{ft} 3 / \mathrm{yr}$ ) for Mississippi River stations and Missouri River stations (red circles) and tributary inputs in the reach from St. Louis, MO, to Louisiana integrated for the period of WY 1990-2013.

Figure 7. Annual water discharge for the three largest flow years in the WY 1990-2013 period for Mississippi River stations at Thebes, IL; Natchez, MS; and Belle Chasse, LA. The "Tarbert Spillway Opening Discharge" line in the Belle Chasse plot refers to the discharge (at Tarbert Landing, MS) that triggers opening of the Bonnet Carre Spillway between Baton Rouge and Belle Chasse, LA, stations. This limits flow at Belle Chasse, $\mathrm{LA}$, to a maximum of 1.25 million $\mathrm{ft}^{3} / \mathrm{sec}$.

Figure 8. Annual average total suspended loads (computed in $10^{6}$ tons/yr) for Mississippi River stations and Missouri River stations (red circles) and tributary inputs in the reach from St. Louis, MO, to Louisiana integrated for the period of WY 1990-2013. Asterisks in the Missouri River refer to insufficient sediment data to calculate a load and in the Red River, Simmesport, LA, and Tarbert Landing, MS, stations refer to incomplete data analysis by the station operator(s). Asterisks at St. Louis, MO, and Chester, MO, refer to the interpretation that the data are over such a limited time frame (WY 2010-2014) that it impacts the load calculated.

Figure 9. Annual average total suspended sand load (computed in $10^{6}$ tons/yr) for Mississippi River stations and Missouri River stations (red circles) and tributary inputs in the reach from St. Louis, MO, to Louisiana integrated for the period of WY 1990-2013. Asterisks in the Missouri River and Kaskaskia River refer to insufficient sediment data to calculate a load and in the Red River, Simmesport, LA, and Tarbert Landing, MS, stations refer to incomplete data analysis by the station operator(s). Asterisks at St. Louis, MO, and Chester, MO, refer to the interpretation that the data are over such a limited time frame (WY 2010-2014) that it impacts the load calculated.

Figure 10. Sediment ratings curve for the Vicksburg, MS, stations on the Mississippi River operated by the USACE Vicksburg District and USGS showing the higher apparent sand (lower plot) attributed to the heavier D96/D99 sampler utilized. quadratic best fit equations utilized for both data sets.

Figure 11. Annual total suspended sediment discharge for the three largest sediment years in the WY 1990-2013 period for Mississippi River stations at Thebes, IL; Natchez, MS; and Belle Chasse, LA. 
Figure 12. Annual total suspended sediment discharge calculated for each year in the WY 1990-2013 period for Mississippi River stations at Thebes, IL; Natchez, MS; and Belle Chasse, LA.

Figure 13. Bar chart of total annual average suspended load in WY 1990-2013 divided into mud and sand fraction for tributaries entering the Mississippi River between St. Louis, MO, and Old River Control.

Figure 14. Pie chart of total annual average suspended load in WY 1990-2013 of tributaries divided into mud and sand fraction and plotted as percentage of the overall tributary sediment flux into the Mississippi River between St. Louis, MO, and Old River Control.

Figure 15. Annual average total suspended load (computed in $10^{6}$ tons/yr) for Mississippi River stations and Missouri River stations (red circles) and tributary inputs in the reach from St. Louis, MO, to Louisiana integrated for the periods of WY 1970-1978 (left; from Keown et al. 1981) and 1990-2013 (right). Asterisks in the Keown data (identified in that study) refer to small sample sets that make the calculated load only a first-order estimate.

Figure 16. Bar chart of total annual average suspended load in WY 1970-1978 (from Keown et al. 1981) and WY 1990-2013 for tributaries entering the Mississippi River between St. Louis, MO, and Old River Control.

Figure 17. Pie chart of total annual average suspended load in WY 1970-1978 (from Keown et al. 1981) and WY 1990-2013 of overall tributary sediment flux into the Mississippi River between St. Louis, MO, and Old River Control.

Figure 18. Annual average total suspended load (computed in $10^{6}$ tons/yr) for Mississippi River stations and Missouri River stations (red circles) and tributary inputs in the reach from St. Louis, MO, to Louisiana integrated for the periods of pre-1953 (left) and WY 1970-1978 (from Keown et al. 1981). Asterisks in the Keown data (identified in that study) refer to small sample sets that make the calculated load only a first-order estimate. Note that Old River Control did not exist in pre-1953, and sediment flux going down the Atchafalaya River pathway was unregulated discharge from the Mississippi River plus flow from the Red River..

\section{Tables}

Table 1. Summary Characteristics for USGS/USACE monitoring stations in the MississippiAtchafalaya River in WY 1990-2013.

Table 2. Summary Characteristics for USGS/USACE monitoring stations in Mississippi River tributaries in WY 1990-2013.

Table 3. Water and sediment discharge in WY1990-2013 for USGS/USACE Mississippi River monitoring stations.

Table 4. Water and sediment discharge in WY1990-2013 for USGS/USACE monitoring stations in Mississippi River tributaries

Table 5. Water budget of Mississippi River from St. Louis, MO, to Belle Chasse, LA, for average annual flow from WY 1990-2013.

Table 6. Total suspended sediment budget of Mississippi River from St. Louis, MO, to Belle Chasse, LA, for average annual flow from WY 1990-2013. 30

Table 7. Suspended sand budget of Mississippi River from St. Louis, MO, to Belle Chasse, 


\section{Preface}

The research documented in this report was conducted as part of the Mississippi River Geomorphology and Potamology (MRG\&P) Program, "Geomorphic Assessments." The MRG\&P Program is sponsored by Headquarters, U.S. Army Corps of Engineers (USACE), and is managed by the USACE Mississippi Valley Division (MVD) in Vicksburg, MS. The MRG\&P Technical Director was Dr. Ty V. Wamsley, and the Program Manager was Mr. Freddie Pinkard. The MVD Commander was MG Michael C. Wehr. The MVD Director of Programs was Mr. Jim Bodron.

The Mississippi River Commission provided Mississippi River engineering direction and policy advice. The Commission members were MG Michael C. Wehr, USACE, President; the Honorable Sam E. Angel; the Honorable R. D. James; the Honorable Norma Jean Mattei, Ph.D.; RDML Gerd F. Glang, National Oceanic and Atmospheric Administration; BG Richard G. Kaiser, USACE Ohio River Division; and BG David C. Hill, USACE Southwest Division.

Mr. Jeffrey R. Eckstein was Deputy Director of ERDC CHL, and Mr. José E. Sánchez was Director.

COL Bryan S. Green was the Commander of ERDC, and the Director was Dr. David W. Pittman. 


\section{Unit Conversion Factors}

\begin{tabular}{|l|c|l|}
\hline Multiply & \multicolumn{1}{l|l|}{ By } & To Obtain \\
\hline acres & $4,046.873$ & square meters \\
\hline acre-feet & $1,233.5$ & cubic meters \\
\hline cubic feet & 0.02831685 & cubic meters \\
\hline cubic yards & 0.7645549 & cubic meters \\
\hline feet & 0.3048 & meters \\
\hline microns & $1.0 \mathrm{E}-06$ & meters \\
\hline miles (U.S. statute) & $1,609.347$ & meters \\
\hline square feet & 0.09290304 & square meters \\
\hline square miles & $2.589998 \mathrm{E}+06$ & square meters \\
\hline tons (2,000 pounds, mass) & 907.1847 & kilograms \\
\hline
\end{tabular}




\section{Background}

The development of the Mississippi River and its flood plain for navigation and flood control has been ongoing since the eighteenth century, with the most concerted efforts occurring as a result of the Flood Control Act of 1928 following the Great Flood of 1927. The Mississippi River \& Tributaries Project that was spawned from the Flood Control Act of 1928 has produced a massive, comprehensive system for flood control and channel stabilization that includes levees, channel improvements, and floodways, as well as tributary reservoirs and other basin improvements (Moore 1972). Additionally, the development of the river for dependable navigation has generated a substantial engineering effort in terms of river training structures, meander cutoffs, and dredging. The historical, present-day, and future morphology of the Mississippi River reflects an integration of all these activities and the features they have created combined with natural drivers of channel change and evolution, including floods and droughts, hurricanes, neo-tectonic activity, geologic outcrops, climatic variability, climate change, and sea level rise. Understanding how these various factors affect river morphology and its short- and long-term evolution is a complex challenge that must be addressed by the river engineers and scientists responsible for managing this system for flood control, navigation, and habitat.

With the complex requirements in navigation, flood risk reduction, and environmental restoration, all with multiple stakeholders, future Mississippi River management will require the most advanced knowledge available. The Mississippi River Geomorphology and Potamology (MRG\&P) Program was developed in recognition of this challenge. The MRG\&P is a joint effort of the U.S. Army Corps of Engineers (USACE), St. Louis, Memphis, Vicksburg, and New Orleans Districts, conducted with the oversight of the Mississippi Valley Division and with technical contributions from the U.S. Army Engineer Research and Development Center. The study presented herein is one component of the MRG\&P. 


\section{Objectives of Study}

In 1981, what was then the Waterways Experiment Station of the USACE in Vicksburg, MS, prepared a two-volume report for the USACE New Orleans District entitled Characterization of the Suspended-Sediment Regime and Bed-Material Gradation of the Mississippi River Basin (Keown et al. 1981). This report prepared as part of an earlier manifestation of the USACE Potamology Program, is hereafter referred to as the "Keown Report." As the report states, "cultural impacts over the past two centuries have shaped the current character of the Mississippi main-stem, suspended-sediment regime." Specifically, the report mentions a number of major cultural impacts:

1. Much of the basin that once had been primarily forest and grasslands was turned to cultural activities.

2. The Old River Control Structures became operational in 1963, preventing unregulated flow from the Mississippi to the Red-Atchafalaya system.

3. Sediment Retention Structures were constructed (1953-1967) and channel improvement features were placed on the Missouri River and its tributaries.

4. Sediment Retention Structures were constructed (1963-1970) and channel improvement features were placed on the Arkansas River and its tributaries.

In addition, improved land use management practices and the placement of numerous stream bank protection works and sediment-retention structures on high-order streams throughout the Mississippi River Basin have undoubtedly reduced main-stem suspended-sediment loads, although these impacts are difficult to quantitatively assess.

The Keown report then proceeds to compile and calculate suspended sediment loads for main-stem Mississippi River gaging stations and tributaries below St. Louis, MO, to the Gulf of Mexico, with Tarbert Landing, MS, being the most downstream record on the Mississippi River and Simmesport, LA, on the Atchafalaya River. The scattered early sediment records were grouped into an examination of the pre-1953 sediment budget and "current" Mississippi River suspended sediment loads-using records from 1970-1978. The primary goal of the suspended sediment load examination (the bed-material load gradation effort in the Keown Report is unrelated to the goals of the present report) was to 
quantify the reductions in Mississippi River main-stem sediment between these periods and to assess the importance of the cultural activities mentioned above.

The present report was conceived as a re-examination of the sediment budget of the main-stem Mississippi River and its tributaries in the intervening $\sim 40$ years since the 1970-1978 compilation, wherever possible utilizing the same gaging stations as the Keown report. The justification for this re-examination is due to several cultural (e.g., anthropogenic) factors:

1. Additional channel retention features have been constructed since the 1970s on major (e.g., lock and dams on the Arkansas and Ohio Rivers) and minor (e.g., reservoirs) tributaries.

2. Additional reaches of the main stem have seen emplacement of bank armoring (e.g., concrete mats) since the 1970 s to limit lateral channel migration.

3. Changes in land use and soil conservation in the Mississippi River Basin have been ongoing.

In addition to anthropogenic alterations to the channel and basin tributary networks that may impact suspended sediment throughput, large floods on the Mississippi River not only impact the annual suspended sediment load in the year they occur but can also have a lasting geomorphic impact by altering channel geometries and the availability of floodplain sediments for denudation, impacting Mississippi River suspended sediment loads for years afterward (see Horowitz 2010). Notably, large Mississippi River floods occurred in 1973, 1983, 1993, 1997, 2008, and 2011.

A final set of factors related to monitoring network adjustments since the Keown report warrants the present re-examination of the Mississippi River suspended sediment budget:

1. The suspended sediment gaging station network on the Mississippi River and its tributaries has evolved since the 1970-1978 period, with new stations coming online and a loss of some stations from the Keown report era.

2. Technologies for measuring water discharge and suspended sediment load at main stem and tributary stations have been improved continuously and today include the use of acoustic methods (e.g., acoustic Doppler current profilers $[\mathrm{ADCP}])$ for measuring current velocities and water discharge and 
depth-integrative, isokinetic suspended sediment samplers (e.g., D9o series; Szalona [1982]). Detailed examinations of state-of-the-art measurement and sampling methodologies that are generally applied on the Mississippi River and its tributaries can be found in Edwards and Glysson (1999), Rasmussen et al. (2009), and Mueller and Wagner (2009).

Variations in sampling methodology and location of stations complicate the comparison with earlier sediment budgets for the Mississippi River Basin from the Keown report. A full assessment of the impacts of these variables on suspended sediment loads at individual gaging stations and the overall sediment budget is incomplete at the time of this report and will be covered in a future MRG\&P report. Hence, the present report will focus on a documentation of changes between the 1970-1978 status report and the present (e.g., 1990-2013) Mississippi River sediment budget. However, the data analysis conducted for the present report revealed the need for two secondary objectives:

Documentation of how variations in suspended sediment sampling field and laboratory procedures through time at a single station, and between stations, affect the suspended sediment load calculation. The latter tends to take place because of a station-to-station difference in the Federal entity conducting the measurements (e.g., USACE, U.S. Geological Survey [USGS]) or because the Mississippi River sampling responsibility crosses unit boundaries (e.g., USACE districts). Errors in laboratory methodology were also identified. The present report will focus on providing examples of the impact of these factors, with a full documentation of all variations at each station to be provided in a future MRG\&P report.

Documenting reach-scale channel increases or decreases in suspended sediment load take place, which is a necessary first step in ascribing anthropogenic or natural causality, is hampered by the absence of gaging stations at critical junctures in the channel. The present report will make suggestions about possible station additions to the Mississippi River monitoring network. The purpose of this objective is to provide a starting point for discussion by the monitoring entities in USGS and USACE about network modification. 


\section{Methodology: Suspended Sediment Discharge Analysis}

\subsection{Data sources}

Data on water and sediment discharge for the present analysis was collected for the period of water years (a water year is 1 October to 30 September) 1990 to 2013 (1 October 1989 to 30 September 2013) by the USGS and USACE at monitoring stations operated on the Mississippi River and its tributaries. These stations are a combination of fixed sensors and boatbased studies at river cross sections located at the monitoring site. The only fixed sensor utilized in the present analysis from these stations was a river or tributary stage (water level) gage. These gages are surveyed into a fixed vertical datum (e.g., MSL, NAVD88) and measure the degree of rise and fall of river stage (in feet) with local changes in river discharge $\left(\mathrm{Q}_{\mathrm{w}}\right.$ in cubic feet per second). Boat-based measurements are taken at intervals of weeks to months by the station operator (USGS or USACE) to (1) directly measure cross-sectionally and depth-integrated water discharge at the monitoring station to calibrate a stage-discharge ratings curve relationship at the site for arriving at daily water discharge from the stage gage measurements, and (2) to collect river suspended sediment concentration measurements (in milligrams) for developing a sediment discharge ( $Q_{s}$ in tons) for each day when integrated with water discharge.

The bulk of the sites utilized for the present analysis were USGS stations, and data were obtained from the online National Water Information System (NWIS) at http://waterdata.usgs.gov/. All stations were utilized with sufficient data to calculate a water and sediment discharge for water years (WY) 19902013 from the reach of Mississippi River between St. Louis, MO, and the Old River Control (Louisiana) division of the river into the Mississippi River and Atchafalaya distributaries. Several stations below Old River Control were selected on the Mississippi River (e.g., Tarbert Landing, MS; Baton Rouge and Belle Chasse, LA) and Atchafalaya (Old River Outflow Channel and Simmesport, LA) to facilitate comparison of results with other recent studies of the river's sediment flux in this region (Allison et al. 2012; Little and Biedenharn 2014). Three additional Mississippi River stations in the reach below St. Louis (e.g., Arkansas City, AR; Vicksburg and Natchez, MS) were also analyzed utilizing data collected by the USACE and either available online from http://rivergages.mvr.usace.army.mil/ (water stage) or obtained 
directly from USACE Vicksburg District personnel (water discharge and suspended sediment data).

Tributaries input to the river in the reach from the region immediately upriver of St. Louis (e.g., Missouri River, Upper Mississippi River, and Illinois River tributaries) to Old River Control were selected if they had been surveyed by Keown et al. (1981) in their analysis of the river's suspended sediment flux. The 17 tributaries selected tend to be the largest in terms of water flux into the Mississippi River. Given that these larger tributaries have multiple monitoring stations along their length, the present study generally selected only the most downstream station prior to their discharging into the Mississippi River where a sufficient water and sediment record existed to perform the calculations. This was done to minimize the additional flux into the tributary in the reach between the monitoring station and the Mississippi River. Wherever possible, stations were also selected for a tributary that had also been chosen by Keown et al. (1981).

The station utilized in Keown et al. (1981) to measure fluxes from the Upper Mississippi River (Hannibal, MO) was deactivated by the USGS between 1984 and 2011, and no substitute was available. Hence, fluxes from the Upper Mississippi River were estimated by subtracting the Illinois River flux at Valley City, IL, from the flux calculated for the USGS below Grafton, IL (USGS 05587455), and Grafton, IL (USGS 05587450), stations immediately below the confluence of both rivers. In the Missouri River tributary, multiple stations were analyzed farther upstream than the last station (Hermann, MO) to examine the relative importance of flux coming out of the dammed Upper Missouri River (the most upstream station analyzed at Yankton, SD, is immediately downstream of the final dam on the river (e.g., Gavins Point Dam) versus the impact of large secondary tributaries (e.g., Kansas, Platte, Grand, Big Sioux) entering the Lower Missouri River. Measuring fluxes from the St. Francis River in Arkansas required combining the results of two stations-Parkin and Madison, AR-to obtain both water and sediment fluxes). The location of all stations utilized in the present study is shown in Figures 1-3. The characteristics of all Mississippi River stations utilized are reported in Table 1, and tributary station information is provided in Table 2. 
Figure 1. Map of the location of Mississippi River stations from St. Louis, MO, to the Gulf of Mexico utilized in the present analysis.

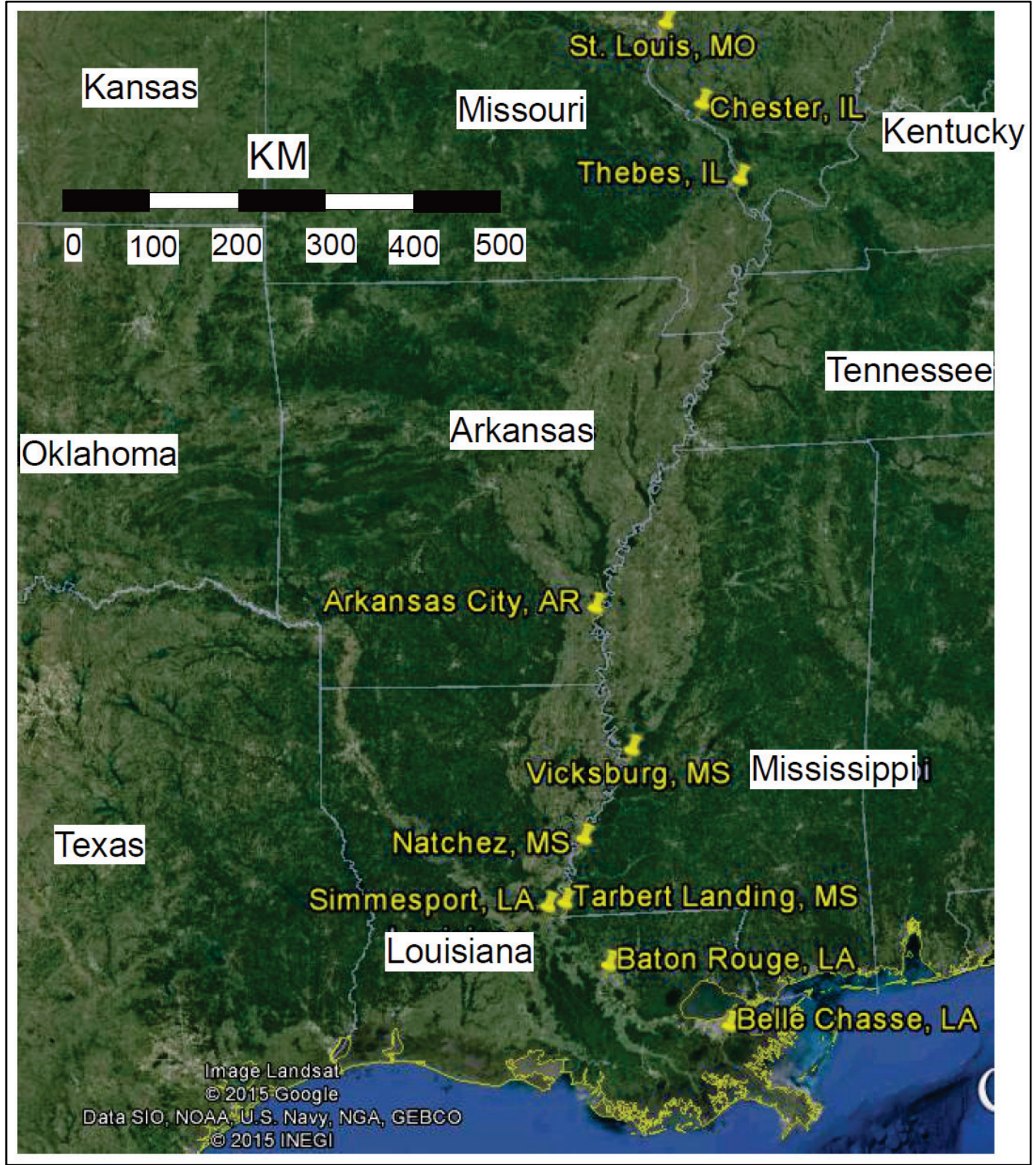


Figure 2. Map of the location of Mississippi River tributary stations north of St. Louis, MO, utilized in the present analysis.

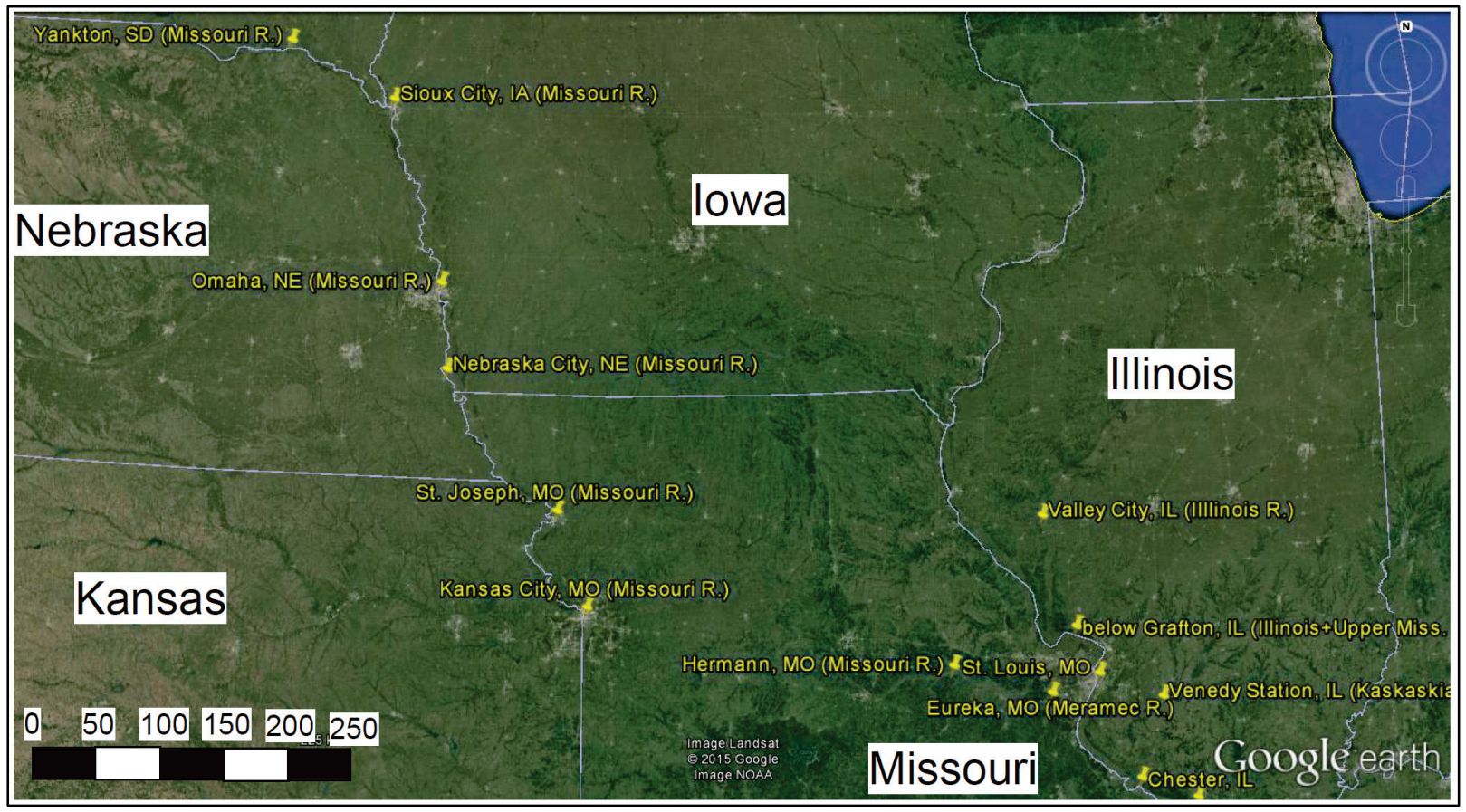

Figure 3. Map of the location of Mississippi River tributary stations south of St. Louis, MO, utilized in the present analysis.

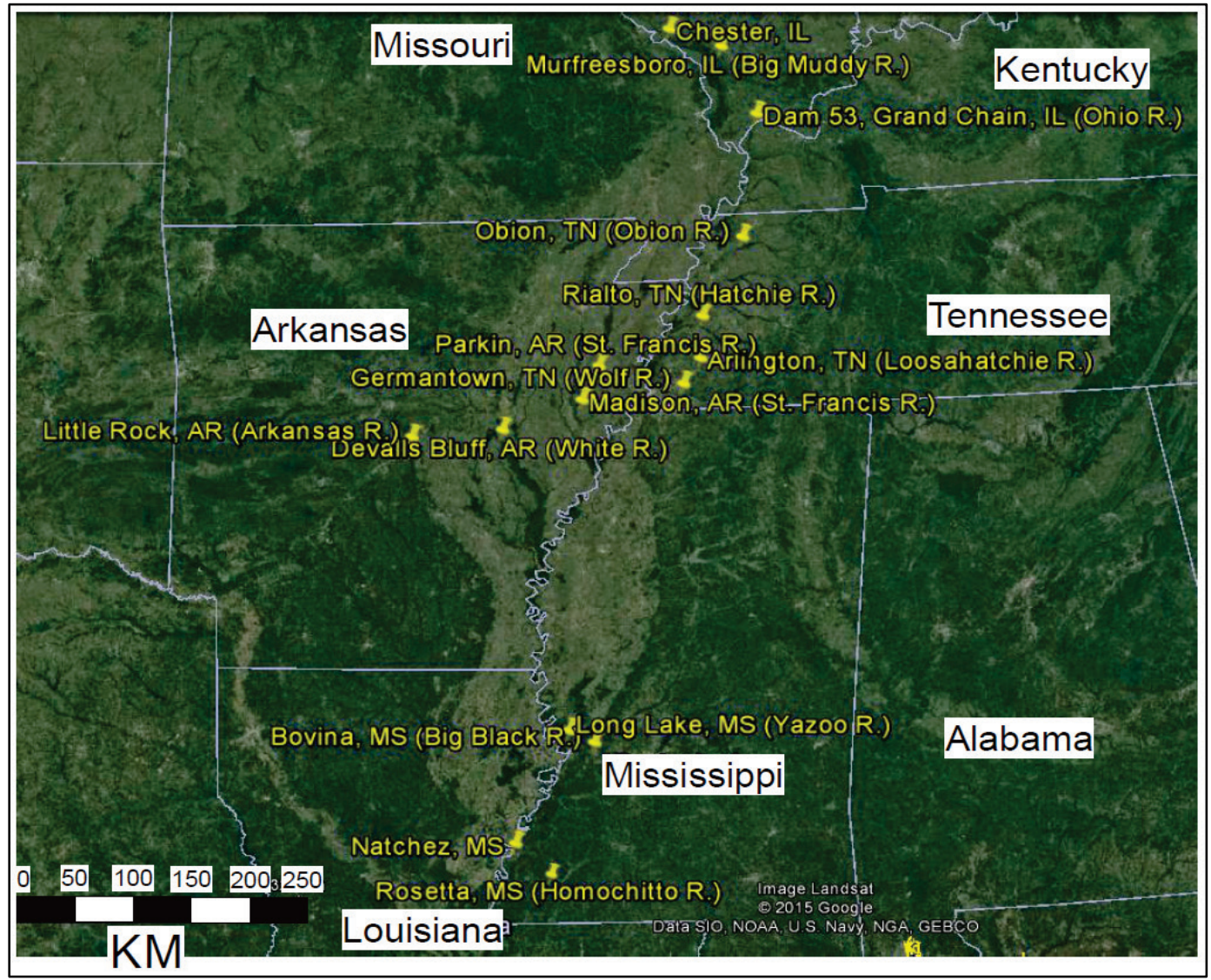


Table 1. Summary Characteristics for USGS/USACE monitoring stations in the Mississippi-Atchafalaya River in WY 1990-2013.

\begin{tabular}{|c|c|c|c|c|c|c|c|c|}
\hline Station Name & Station Identifier & $\begin{array}{l}\text { Period of } \\
\text { Suspended } \\
\text { Sediment Record }\end{array}$ & $\begin{array}{l}\text { Period of } \\
\text { Stream Flow } \\
\text { Record }\end{array}$ & $\begin{array}{l}\text { Number of } \\
\text { Utilized Total } \\
\text { (sand) } \\
\text { Suspended } \\
\text { Load }\end{array}$ & $\begin{array}{l}\text { Sampler** } \\
\text { Type(s) }\end{array}$ & $\begin{array}{l}\text { Highest } \\
\text { Observed } \\
\text { Stream Flow } \\
\text { (ft } 3 / \mathrm{sec} \text { ) in } \\
\text { Sediment } \\
\text { Record }\end{array}$ & $\begin{array}{l}\text { Highest } \\
\text { Observed } \\
\text { Stream Flow } \\
\left(\mathrm{ft}^{3} / \mathrm{sec}\right) \text { in } \\
1990-2013\end{array}$ & $\begin{array}{l}\text { Best Fit } \\
\text { Total (sand) } \\
\text { Ratings } \\
\text { Curve } \\
\text { Applied* }\end{array}$ \\
\hline $\begin{array}{l}\text { Grafton, IL/ } \\
\text { Below Grafton } \\
\text { (Upper } \\
\text { Miss+Illinois) }\end{array}$ & \begin{tabular}{|l} 
USGS \\
05587455 \\
USGS05587450
\end{tabular} & $\begin{array}{l}\text { 1990-2002 } \\
\text { 2009-2014 } \\
\text { Below Gr. }\end{array}$ & $\begin{array}{l}\text { 1990-2013 } \\
\text { Grafton }\end{array}$ & 415 (158) & $\begin{array}{l}\text { ? (to 2007) } \\
\text { D-96 (2008- } \\
2013)\end{array}$ & 491,000 & $\begin{array}{l}596,000 \\
(1993)\end{array}$ & $S(L)$ \\
\hline St. Louis, MO & \begin{tabular}{|l} 
USGS \\
07010000
\end{tabular} & 2010-2014 & $1990-2013$ & $39(12)$ & D-96 & 790,000 & $\begin{array}{l}1,050,000 \\
(1993)\end{array}$ & $\mathrm{Q}(\mathrm{Q})$ \\
\hline Chester, IL & \begin{tabular}{|l} 
USGS \\
07020500
\end{tabular} & 2010-2014 & $1990-2013$ & 49 (12) & D-96 & 795,000 & $\begin{array}{l}1,000,000 \\
(1993)\end{array}$ & $\mathrm{Q}(\mathrm{Q})$ \\
\hline Thebes, IL & $\begin{array}{l}\text { USGS } \\
07022000\end{array}$ & \begin{tabular}{|l}
$1990-1992$ \\
$1995-2013$
\end{tabular} & 1990-2013 & $278(254)$ & $\begin{array}{l}\text { ? (to 2007) } \\
\text { D-96 } \\
\text { (2008-2013) }\end{array}$ & 771,000 & $\begin{array}{l}978,000 \\
(1993)\end{array}$ & $S(Q)$ \\
\hline Arkansas City, AR & USACE MVK & $\begin{array}{l}\text { 1990, } 1997 \\
1999-2011\end{array}$ & $1990-2013$ & $82(82)$ & P-61 & $1,249,000$ & $\begin{array}{l}2,290,000 \\
(2011)\end{array}$ & $\mathrm{Q}(\mathrm{Q})$ \\
\hline Vicksburg, MS & USACE MVK & $\begin{array}{l}1990, \\
1997-2014\end{array}$ & $1990-2013$ & $86(86)$ & P-61 & $1,769,000$ & $\begin{array}{l}2,300,000 \\
(2011)\end{array}$ & $S(S)$ \\
\hline Natchez, MS & USACE MVK & \begin{tabular}{|l|}
1990, \\
$1999-2014$
\end{tabular} & $1990-2013$ & $98(98)$ & P-61 & $1,431,000$ & $\begin{array}{l}2,220,000 \\
(2011)\end{array}$ & $S(S)$ \\
\hline $\begin{array}{l}\text { Tarbert Landing, } \\
\text { MS }\end{array}$ & \begin{tabular}{|l|} 
USGS \\
07295100 \\
USACE 01100
\end{tabular} & not done & $1990-2013$ & not done & not done & not done & $\begin{array}{l}1,619,000 \\
(2011)\end{array}$ & none \\
\hline $\begin{array}{l}\text { Old River Outfall, } \\
\text { LA }\end{array}$ & \begin{tabular}{|l|} 
USGS \\
07381482 \\
USACE 02600
\end{tabular} & not done & 1990-2013 & not done & not done & not done & $\begin{array}{l}671,000 \\
(2011)\end{array}$ & none \\
\hline Simmesport, LA & \begin{tabular}{|l|} 
USGS \\
O7381490 \\
USACE 03045
\end{tabular} & not done & 1990-2013 & not done & not done & not done & \begin{tabular}{|l}
692,000 \\
$(2011)$
\end{tabular} & none \\
\hline
\end{tabular}




\begin{tabular}{|c|c|c|c|c|c|c|c|c|}
\hline Station Name & Station Identifier & $\begin{array}{l}\text { Period of } \\
\text { Suspended } \\
\text { Sediment Record }\end{array}$ & $\begin{array}{l}\text { Period of } \\
\text { Stream Flow } \\
\text { Record }\end{array}$ & $\begin{array}{l}\text { Number of } \\
\text { Utilized Total } \\
\text { (sand) } \\
\text { Suspended } \\
\text { Load }\end{array}$ & $\begin{array}{l}\text { Sampler** } \\
\text { Type(s) }\end{array}$ & $\begin{array}{l}\text { Highest } \\
\text { Observed } \\
\text { Stream Flow } \\
\left(\mathrm{ft}^{3} / \mathrm{sec} \text { ) in }\right. \\
\text { Sediment } \\
\text { Record }\end{array}$ & $\begin{array}{l}\text { Highest } \\
\text { Observed } \\
\text { Stream Flow } \\
\left(\mathrm{ft}^{3} / \mathrm{sec}\right) \text { in } \\
1990-2013\end{array}$ & $\begin{array}{l}\text { Best Fit } \\
\text { Total (sand) } \\
\text { Ratings } \\
\text { Curve } \\
\text { Applied* }\end{array}$ \\
\hline Baton Rouge, LA & $\begin{array}{l}\text { USGS } \\
07374000\end{array}$ & $\begin{array}{l}2004-2005 \\
2007-2014\end{array}$ & $\begin{array}{l}\text { 1990-1995, } \\
\text { 1998-2013 }\end{array}$ & $120(117)$ & D-96/99 & $1,429,000$ & $\begin{array}{l}1,429,000 \\
(2011)\end{array}$ & $S(S)$ \\
\hline Belle Chasse, LA & $\begin{array}{l}\text { USGS } \\
07374525\end{array}$ & $\begin{array}{l}1990-1997 \\
2007-2014\end{array}$ & $1990-2013$ & $138(125)$ & $\begin{array}{l}\text { P-63 (to 1997) } \\
\text { D-96/99 } \\
(2006-2014)\end{array}$ & $1,190,000$ & $\begin{array}{l}1,277,455 \\
(1998)\end{array}$ & $\mathrm{Q}(\mathrm{S})$ \\
\hline
\end{tabular}

${ }^{\star} \mathrm{Q}=$ quadratic, $\mathrm{S}=$ three parameter sigmoidal

$* *$

D-74 = Depth integrating suspended hand line sampler (62 pounds [lb])

D-77 = Depth integrating suspended sampler $(90 \mathrm{lb})$

D-95 = Depth integrating suspended sampler $(29 \mathrm{lb})$

D-96 = Depth integrating suspended sampler (132 lb)

D-99 = Depth integrating suspended sampler (285 lb)

DH-2 = Bag-type suspended sediment/water quality sampler (30 lb)

DH-48 = Depth integrating suspended wading type sampler (3.5 lb)

DH-59 = Depth integrating suspended hand line sampler (22 lb)

DH-76 = Depth integrating suspended hand line sampler (25lb)

P-61 = Point integrating suspended sediment sampler (105 lb)

P-63 = Point integrating suspended sediment sampler (202 lb)

WB $=$ Weighted bottle (various weights) 
Table 2. Summary Characteristics for USGS/USACE monitoring stations in Mississippi River tributaries in WY 1990-2013.

\begin{tabular}{|c|c|c|c|c|c|c|c|c|}
\hline Station Name & Station Identifier & $\begin{array}{l}\text { Period of } \\
\text { Suspended } \\
\text { Sediment } \\
\text { Record }\end{array}$ & $\begin{array}{l}\text { Period of } \\
\text { Stream Flow } \\
\text { Record }\end{array}$ & \begin{tabular}{|l|} 
Number of \\
Utilized \\
Total \\
(sand) \\
Suspended \\
Load
\end{tabular} & Sampler Type(s) & $\begin{array}{l}\text { Highest Observed } \\
\text { Stream Flow } \\
\left(\mathrm{ft}^{3} / \mathrm{sec}\right) \text { in } \\
\text { Sediment Record }\end{array}$ & $\begin{array}{l}\text { Highest } \\
\text { Observed } \\
\text { Stream Flow } \\
\text { (ft } 3 / \text { sec) in } \\
1990-2013\end{array}$ & $\begin{array}{l}\text { Best Fit } \\
\text { Total } \\
\text { (sand) } \\
\text { Ratings } \\
\text { Curve } \\
\text { Applied* }\end{array}$ \\
\hline $\begin{array}{l}\text { Missouri } \\
\text { (Yankton, SD) }\end{array}$ & USGS 06467500 & $2000-2008$ & $1990-2013$ & $57(45)$ & \begin{tabular}{|l|} 
P-61/63 (to 2003) \\
D-95 (2003- \\
$2008)$
\end{tabular} & 33,000 & \begin{tabular}{|l|}
202,626 \\
$(2011)$
\end{tabular} & $P(Q)$ \\
\hline $\begin{array}{l}\text { Missouri } \\
\text { (Sioux City, IA) }\end{array}$ & USGS 06486000 & Limited data & 1990-2013 & none & P-61 & none & & none \\
\hline $\begin{array}{l}\text { Missouri } \\
\text { (Omaha, NE) }\end{array}$ & USGS 06610000 & Limited data & $1990-2013$ & none & P-61 & none & & none \\
\hline $\begin{array}{l}\text { Missouri } \\
\text { (Nebraska City, NE) }\end{array}$ & USGS 06807000 & Limited data & $1990-2013$ & none & P-61 & none & & none \\
\hline $\begin{array}{l}\text { Missouri } \\
\text { (St. Joseph, MO) }\end{array}$ & USGS 06818000 & $1990-2008$ & $1990-2013$ & $163(140)$ & P-61 & 146,000 & $\begin{array}{l}146,000 \\
(1993, \\
2011) \\
\end{array}$ & $\mathrm{S}(\mathrm{Q})$ \\
\hline $\begin{array}{l}\text { Missouri } \\
\text { (Kansas City, MO) }\end{array}$ & USGS 06893000 & 1990-2008 & 1990-2013 & $160(130)$ & P-61 & 167,000 & $\begin{array}{l}529,000 \\
(1993)\end{array}$ & $\mathrm{S}(\mathrm{Q})$ \\
\hline $\begin{array}{l}\text { Missouri } \\
\text { (Hermann, MO) }\end{array}$ & USGS 0693450 & 1990-2009 & $1990-2013$ & 342 (318) & $\begin{array}{l}\text { P-61/63 (to 2003) } \\
\text { D-96 (2004-2014) }\end{array}$ & 716,000 & $\begin{array}{l}739,000 \\
(1993)\end{array}$ & $\mathrm{S}(\mathrm{S})$ \\
\hline $\begin{array}{l}\text { Illinois } \\
\text { (Valley City, IL) }\end{array}$ & USGS 05586100 & 1990-2013 & 1990-2013 & 499 (183) & \begin{tabular}{|l|} 
P-61A/DH-2 \\
$(1990-1995)$ \\
D-77/WB \\
$(1996-2002$ \\
DH-22/WB \\
$(2003-2013)$
\end{tabular} & 111,000 & $\begin{array}{l}111,000 \\
(2013)\end{array}$ & $\mathrm{S}(\mathrm{S})$ \\
\hline $\begin{array}{l}\text { Meramec } \\
\text { (Eureka, MO) }\end{array}$ & USGS 07019000 & 1986-1994 & 1990-2013 & $53(44)$ & ? & 43,900 & \begin{tabular}{|l|}
118,000 \\
$(1994)$
\end{tabular} & $\mathrm{Q}(\mathrm{Q})$ \\
\hline
\end{tabular}




\begin{tabular}{|c|c|c|c|c|c|c|c|c|}
\hline Station Name & Station Identifier & $\begin{array}{l}\text { Period of } \\
\text { Suspended } \\
\text { Sediment } \\
\text { Record }\end{array}$ & $\begin{array}{l}\text { Period of } \\
\text { Stream Flow } \\
\text { Record }\end{array}$ & $\begin{array}{l}\text { Number of } \\
\text { Utilized } \\
\text { Total } \\
\text { (sand) } \\
\text { Suspended } \\
\text { Load }\end{array}$ & Sampler Type(s) & $\begin{array}{l}\text { Highest Observed } \\
\text { Stream Flow } \\
\left(\mathrm{ft}^{3} / \mathrm{sec}\right) \text { in } \\
\text { Sediment Record }\end{array}$ & $\begin{array}{l}\text { Highest } \\
\text { Observed } \\
\text { Stream Flow } \\
\left(\mathrm{ft}^{3} / \mathrm{sec}\right) \text { in } \\
1990-2013\end{array}$ & $\begin{array}{l}\text { Best Fit } \\
\text { Total } \\
\text { (sand) } \\
\text { Ratings } \\
\text { Curve } \\
\text { Applied* }\end{array}$ \\
\hline $\begin{array}{l}\text { Kaskaskia } \\
\text { (Venedy Station, IL) }\end{array}$ & USGS 05594100 & 1990-1992 & $\begin{array}{l}1990-1995, \\
1998-2013\end{array}$ & $38(21)$ & $?$ & 10,900 & $\begin{array}{l}48,700 \\
(1995)\end{array}$ & $\mathrm{Q}(\mathrm{Q})$ \\
\hline $\begin{array}{l}\text { Big Muddy } \\
\text { (Murfreesboro, IL) }\end{array}$ & USGS 05599500 & 1990-1992 & $1990-2013$ & $111(30)$ & $\begin{array}{l}\mathrm{DH}-76 \\
(1990-1992)\end{array}$ & 14,600 & $\begin{array}{l}38,200 \\
(2011)\end{array}$ & $L(Q)$ \\
\hline $\begin{array}{l}\text { Ohio (Dam } 53 \text { near } \\
\text { Grand Chain, IL) }\end{array}$ & USGS 03612500 & $1990-2013$ & 1990-2013 & 309 (262) & $\begin{array}{l}\text { D-74 (1990-2002) } \\
\text { D-96 (2003-2013) }\end{array}$ & $1,260,000$ & $\begin{array}{l}1,260,000 \\
(2011)\end{array}$ & $\mathrm{Q}(\mathrm{Q})$ \\
\hline $\begin{array}{l}\text { Obion } \\
\text { (Obion, TN) }\end{array}$ & USGS 07026040 & 1990-1995 & $\begin{array}{l}1990-1995 \\
2002-2013\end{array}$ & $17(16)$ & $?$ & 5,860 & $\begin{array}{l}63,900 \\
(2011)\end{array}$ & P3 (Q) \\
\hline $\begin{array}{l}\text { Hatchie } \\
\text { (Rialto, TN) }\end{array}$ & USGS 07030050 & 2004-2008 & 2004-2013 & $22(4)$ & DH-59 & 7,290 & $\begin{array}{l}53,000 \\
(2011)\end{array}$ & $\mathrm{S}(\mathrm{Q})$ \\
\hline $\begin{array}{l}\text { Loosahatchie } \\
\text { (Arlington, TN) }\end{array}$ & USGS 07030240 & $1979-1982$ & $1990-2013$ & $32(3)$ & $?$ & 2,650 & $\begin{array}{l}26,000 \\
(2010)\end{array}$ & $\mathrm{Q}$ (none) \\
\hline $\begin{array}{l}\text { Wolf } \\
\text { (Germantown, TN) }\end{array}$ & USGS 07031650 & $\begin{array}{l}1979-1986 \\
2001-2005\end{array}$ & 1991-2013 & $55(8)$ & $?$ & 8,580 & $\begin{array}{l}26,700 \\
(2011)\end{array}$ & $E G(Q)$ \\
\hline $\begin{array}{l}\text { St. Francis } \\
\text { (Parkin/Madison, } \\
\text { AR) }\end{array}$ & $\begin{array}{l}\text { USGS } 07047800 \\
\text { USGS } 07047907\end{array}$ & $\begin{array}{l}1990-2009 \\
\text { (combined) }\end{array}$ & $\begin{array}{l}1990-1994, \\
1998-2002, \\
2005-2010 \\
\text { (Parkin) }\end{array}$ & $264(28)$ & $\begin{array}{l}\text { DH-59 (Madison) } \\
\text { ? (Parkin) }\end{array}$ & $\begin{array}{l}39,300 \\
\text { (Madison) }\end{array}$ & $\begin{array}{l}16,600 \\
(2006) \\
\text { (Parkin) }\end{array}$ & $\mathrm{Q}(\mathrm{Q})$ \\
\hline $\begin{array}{l}\text { White } \\
\text { (Devall's Bluff, AR) }\end{array}$ & USGS 07077000 & 2001-2012 & $1990-2013$ & $64(63)$ & $\begin{array}{l}\text { D-96/D95 } \\
\text { 2001-2013 }\end{array}$ & 92,900 & $\begin{array}{l}187,000 \\
(2008)\end{array}$ & $E G(P 2)$ \\
\hline $\begin{array}{l}\text { Arkansas (Terry Lock } \\
\text { \& Dam below Little } \\
\text { Rock, AR) }\end{array}$ & USGS 07263620 & $1990-2014$ & $1990-2013$ & 211 (196) & $\begin{array}{l}\text { WB/D-96 } \\
(1995-2013)\end{array}$ & 252,000 & $\begin{array}{l}404,000 \\
(1990)\end{array}$ & $\mathrm{Q}(\mathrm{Q})$ \\
\hline
\end{tabular}




\begin{tabular}{|c|c|c|c|c|c|c|c|c|}
\hline Station Name & Station Identifier & $\begin{array}{l}\text { Period of } \\
\text { Suspended } \\
\text { Sediment } \\
\text { Record }\end{array}$ & $\begin{array}{l}\text { Period of } \\
\text { Stream Flow } \\
\text { Record }\end{array}$ & $\begin{array}{l}\text { Number of } \\
\text { Utilized } \\
\text { Total } \\
\text { (sand) } \\
\text { Suspended } \\
\text { Load }\end{array}$ & Sampler Type(s) & $\begin{array}{l}\text { Highest Observed } \\
\text { Stream Flow } \\
\text { ( } \mathrm{ft}^{3} / \mathrm{sec} \text { ) in } \\
\text { Sediment Record }\end{array}$ & $\begin{array}{l}\text { Highest } \\
\text { Observed } \\
\text { Stream Flow } \\
\left(\mathrm{ft}^{3} / \mathrm{sec}\right) \text { in } \\
1990-2013\end{array}$ & $\begin{array}{l}\text { Best Fit } \\
\text { Total } \\
\text { (sand) } \\
\text { Ratings } \\
\text { Curve } \\
\text { Applied* }\end{array}$ \\
\hline $\begin{array}{l}\text { Yazoo } \\
\text { (Long Lake, MS) }\end{array}$ & USGS 07288955 & $1994-2013$ & 1996-2013 & $325(100)$ & $\begin{array}{l}\text { WB 1995-1996 } \\
\text { D-77 1998-2002 } \\
\text { WB/D-96 2003- } \\
2013\end{array}$ & 72,500 & $\begin{array}{l}86,100 \\
(2009)\end{array}$ & $\mathrm{Q}(\mathrm{EG})$ \\
\hline $\begin{array}{l}\text { Big Black } \\
\text { (Bovina, MS) }\end{array}$ & USGS 07290000 & 1990-1994 & $1990-2013$ & $25(15)$ & WB & 21,800 & $\begin{array}{l}53,200 \\
(1991)\end{array}$ & $\mathrm{Q}(\mathrm{EG})$ \\
\hline $\begin{array}{l}\text { Homochitto } \\
\text { (Rosetta, MS) }\end{array}$ & USGS 07292500 & $\begin{array}{l}1990-1994 \\
2011\end{array}$ & $1990-2013$ & $27(19)$ & WB/DH-48/DH-59 & 24,000 & $\begin{array}{l}69,600 \\
(2001)\end{array}$ & $\mathrm{Q}(\mathrm{Q})$ \\
\hline
\end{tabular}

${ }^{*} \mathrm{Q}=$ quadratic, $\mathrm{S}=$ three parameter sigmoidal, $\mathrm{P} 2=$ two parameter power law, $\mathrm{P} 3=$ three parameter power law, $\mathrm{L}=\mathrm{second}$-order logarithm, $\mathrm{EG}=\mathrm{Single}$, threeparameter exponential growth

**

D-74 = Depth integrating suspended hand line sampler (62 lb)

D-77 = Depth integrating suspended sampler (90 lb)

D-95 = Depth integrating suspended sampler (29 lb)

D-96 = Depth integrating suspended sampler (132 lb)

D-99 = Depth integrating suspended sampler (285 lb)

$\mathrm{DH}-2$ = Bag-type suspended sediment/water quality sampler $(30 \mathrm{lb})$

DH-48 = Depth integrating suspended wading type sampler $(3.5 \mathrm{lb})$
P-61 = Point integrating suspended sediment sampler (105 lb) P-63 = Point integrating suspended sediment sampler (202 lb) DH-76 = Depth integrating suspended hand line sampler (25 lb) DH-59 = Depth integrating suspended hand line sampler $(22 \mathrm{lb})$ WB $=$ Weighted bottle (various weights) 


\subsection{Method for calculating water discharge}

The first step in calculating sediment discharge (Qs) for a monitoring station on the Mississippi River or a tributary is to obtain daily water discharge $\left(\mathrm{Q}_{\mathrm{w}}\right)$; this information is utilized to integrate boat-based, suspended sediment concentrations with flow velocities over the entire cross section. For the majority of stations, daily water discharge was available directly from the USGS NWIS website (cited above) or was obtained from USACE Vicksburg District. Qw at these sites since the $1990 \mathrm{~s}$ has been measured directly during weekly-to-monthly, boat-based studies with an ADCP using methods outlined in Mueller and Wagner (2009). These measurements are then utilized by the station operator to construct a water ratings curve with daily stages at the site to develop a daily record of $\mathrm{Q}_{\mathrm{w}}$, which is what is available on the Federal websites. Gaps in the daily stream flow record over the WY 1990-2013 are recorded in Table 1 and 2 and reflect the absence of an operating stage gage during these periods at these sites; partial daily records were removed from the daily record so only complete water years were utilized in the annual sediment discharge calculations. In the case of the stations at Baton Rouge and Belle Chasse, LA, stage gage information was available before the present boat-based operations began in 2005 in Baton Rouge, LA, and 2008 in Belle Chasse, LA (Belle Chasse was also measured for sediment flux between 1977 and 1997). This allowed calculation of a water ratings curve for these two stations prior to boat-based operations beginning by using the stage gage measurements to extend the record. This procedure is instructive in that it follows the procedure utilized by the USGS/USACE operators for the Qw daily data published online. Figure 4 shows the stage-discharge relationship for the period of overlapping boat-based and stage measurements (2005-2013) at the Baton Rouge, LA, monitoring station on the Mississippi River. A quadratic best fit regression was fit to the points and was used to calculate a WY 1990-2004 daily water discharge using the stage measurements. The post-2005 water discharges at Baton Rouge and Belle Chasse, LA, were then derived from daily stage, rather than the daily water flux available online, for consistency. 
Figure 4. Water ratings curve (stage-discharge) for the Mississippi River (USGS) station at Baton Rouge, LA.

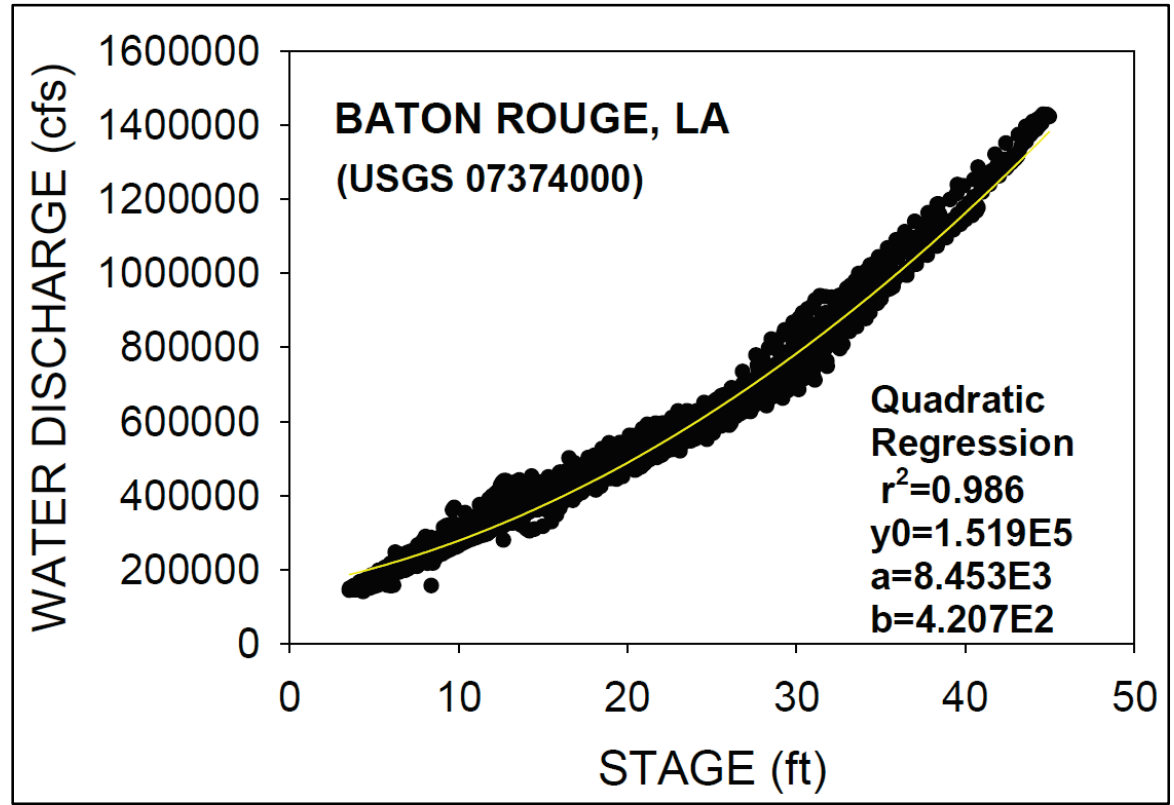

\subsection{Sediment discharge calculations}

Sediment discharge in rivers is typically calculated utilizing a sediment ratings curve methodology (Porterfield 1972). Given the expense and difficulty of daily boat-based measurements to follow variations in the river's suspended sediment load with the rise and fall of the river's water discharge, the ratings curve method provides a water discharge-based methodology for predicting daily suspended sediment load. The ratings curve is constructed (Figure 5) using discrete, boat-based measurements of suspended sediment load at a station. Suspended sediment load is calculated using the equation

$$
Q_{s}=Q_{w} \times C_{s} \times k
$$

where:

$Q s$ = suspended sediment discharge in tons per day

$Q w=$ water discharge in cubic feet per second

$C s=$ mean concentration of suspended sediment in the cross section in milligrams per liter

$k=\mathrm{a}$ coefficient based on the water discharge that assumes a specific weight of 2.65 for sediment. 
Figure 5. Sediment ratings curve for the Mississippi River at Thebes, IL, (total and sand fraction) constructed using boatbased data on suspended sediment load from the USGS NWIS.

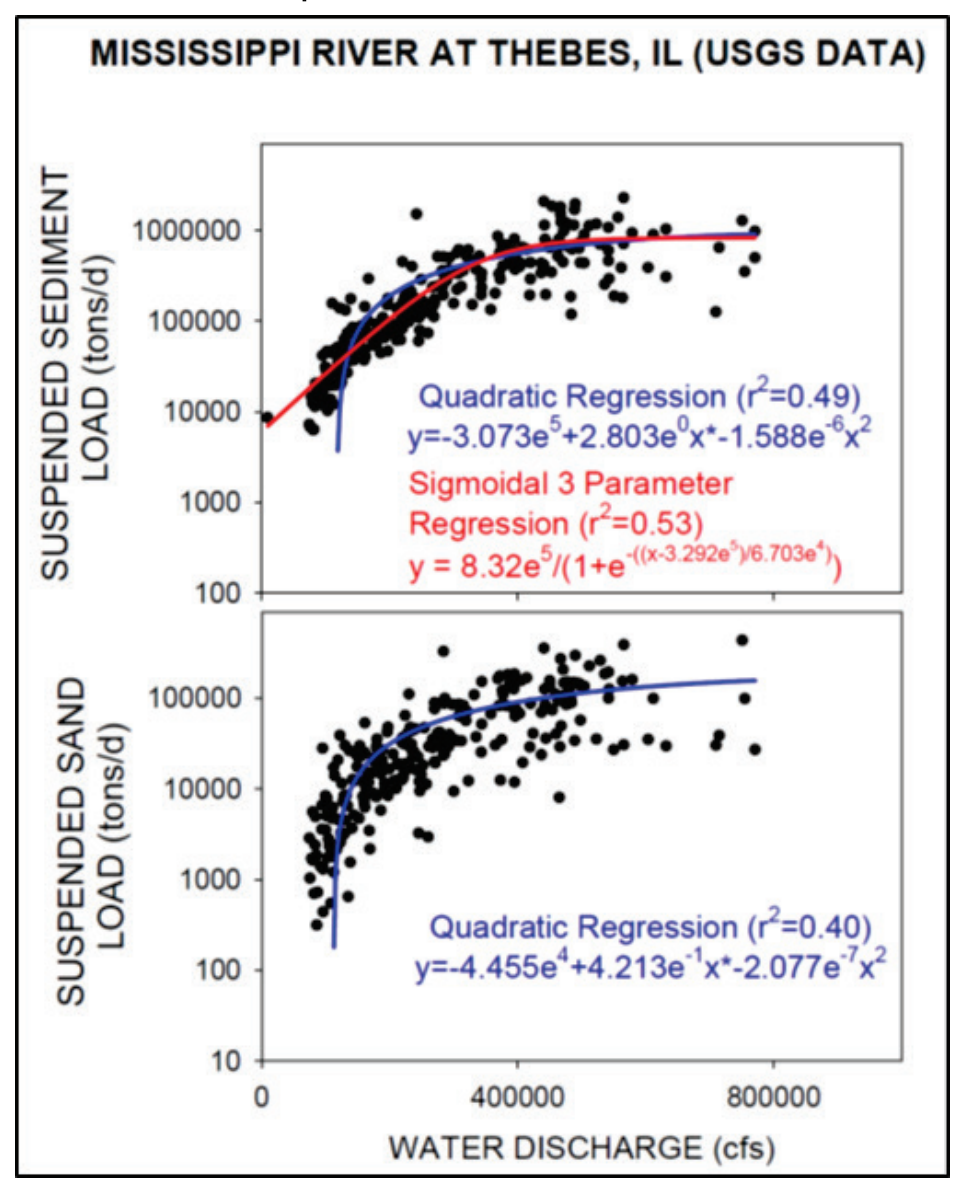

For the present study, discrete boat-based measurements of suspended sediment load were obtained directly from the USGS NWIS website or from USACE Vicksburg District, with Equation 1 calculated by the data collection entity. However, the fundamental methods of computing daily suspended sediment discharge by these entities has not changed since the 1940s (Gray and Simões 2008; Rasmussen et al. 2009) and derives the mean concentration of suspended sediment in the cross section (Cs) for Equation 1 using a flow-weighted interpolation. Typically, a suspended sediment sampler is utilized to collect multiple depth samples (0.1, 0.3, $0.5,0.7$, and 0.9 total water depth is a typical pattern) along a series of verticals (typically $3-8$ ) across the sample transect. As explained in detail in Edwards and Glysson (1999), a mean concentration is then derived for a subsection of the cross section for each vertical (sample point is the centroid) and a water discharge applied (typically measured by ADCP immediately before or after the transect sample collection) using either the equal discharge increment or equal width increment method. The derived 
suspended sediment discharge for each cross-sectional increment is then summed to derive a depth-integrated and cross-sectionally integrated, total suspended sediment discharge for that day. If, in the laboratory, the samples that were measured for suspended concentration (milligrams per liter) were sieved to determine the concentration of sand ( $>62.5$ micron $(\mu \mathrm{m})$ fraction) in the sample, then a separate sand suspended sediment load can be derived and utilized to construct a sediment ratings curve for sand (Figure 5).

All data falling within the WY 1990-2013 time window (1 October 1989 to 30 Sept 2013) were utilized with a few exceptions to calculate daily suspended sediment loads. Inconsistencies in the boat-based suspended load measurements from the three USACE Mississippi River stations (e.g., Arkansas City, AR; Vicksburg and Natchez, MS) including sand loads that exceeded total loads, likely due to (1) record keeping errors, (2) scale weighing errors, or (3) laboratory procedural errors led to the removal of data collected in WY 1991-1996 (Table 1). A number of stations (reported in Tables 1 and 2) utilized data from WY 2014 if available to increase the limited number of measurements. Data from pre-1990 was utilized for three tributaries (e.g., Meramec, Lossahatchie, Wolf; Table 2), even though it complicates comparison with the Keown 1970-1978 data, but without this data, insufficient data would have been available to fit a curve to derive daily suspended sediment loads. Tables 1 and 2 also report the total number of measurements utilized in the curve fitting and the maximum water discharge represented in the available data points to aid in interpreting the "quality" of the resulting load calculation.

The development of a ratings curve for a plot of suspended sediment load versus discharge (Figure 5) has been conducted utilizing many different curve fitting methods, including the use of individual annual curves (Horowitz 2003) and semi-automated procedures (Koltun et al. 1994; Runkel et al. 2004). For the present study, to allow inter-comparison with the author's previous efforts in the Louisiana section of the Mississippi River (Allison et al. 2012; Little and Biedenharn 2014), two methods of curve fitting the data were conducted. As reported in Tables 3 and 4, total and sand-suspended sediment load at all stations were calculated using a quadratic best fit regression following Little and Biedenharn (2014):

$$
Q_{S}=a+b Q_{w}+c Q_{w}^{2}
$$


where:

$Q s=$ total (or sand) load in tons per day

$Q w=$ water discharge in cubic feet per second

$a, b, c=$ quadratic coefficients.

In addition, a "best fit" sediment load was calculated utilizing a series of other curve fits that were shown in Allison et al. (2012) to sometimes provide an $\mathrm{r}^{2}$ better than the quadratic equation. A total of eight additional equations were tested, and the one with the highest $\mathrm{r}^{2}$ is reported in Tables 3 and 4. If the quadratic equation had an equal or higher $\mathrm{r}^{2}$ than all the tested equations, only the quadratic result is reported in Tables 3 and 4. It is this "best fit" calculation, identified in Tables 1 and 2, that is utilized in all subsequent discussions of sediment budget results of the present study. The eight equations are as follows:

Sigmoidal three parameter:

$$
Q_{s}=Y_{0}+\frac{a}{\left(1+e^{-\left(\frac{Q_{w}-X_{0}}{b}\right)}\right)^{c}}
$$

Power Law two parameter:

$$
Q_{S}=a Q_{w}^{b}
$$

Power Law three parameter:

$$
Q_{s}=Y_{0}+a \times Q_{w}^{b}
$$

Second-Order Logarithm:

$$
Q_{s}=Y_{0}+\left(a \times \ln \left(Q_{w}\right)\right)+\left(b \times \ln \left(Q_{w}\right)^{2}\right)
$$

Third-Order Logarithm:

$$
Q_{s}=Y_{0}+\left(a \times \ln \left(Q_{w}\right)\right)+\left(b \times \ln \left(Q_{w}\right)^{2}\right)+\left(c \times \ln \left(Q_{w}\right)^{3}\right)
$$


Exponential Growth (single, three parameter):

$$
Q_{s}=Y_{0}+a \times e^{\left(b Q_{w}\right)}
$$

Exponential Rise to Maximum (single, three parameter):

$$
Q_{s}=Y_{0}+a \times\left(1-e^{\left(-b Q_{w}\right)}\right)
$$

Exponential Rise to Maximum (double, four parameter):

$$
Q_{s}=a \times\left(1-e^{\left(-b Q_{w}\right)}\right)+c \times\left(1-e^{\left(-d Q_{w}\right)}\right)
$$

Station examples of the application of coefficients to the equations and the resulting curve fit for total and sand-sized suspended sediment discharge are shown in Figure 5.

Daily sediment discharges were calculated for each Mississippi River and tributary station using the best fit sediment ratings curve and the daily water discharges between 1 October 1990 and 30 September 2013. The resultant daily values were summed to derive an annual suspended sediment load for each WY. Annual values were then averaged to arrive at a mean annual sediment load for each station over that $24 \mathrm{yr}$ period. Tables 3 and 4 report these values for Mississippi River and tributary stations, respectively, and also report the maximum and minimum annual suspended sediment load during WY 1990-2013.

\subsection{Sediment discharge interpretation}

While the creation of daily, annual, and mean WY1990-2013, total and sand-suspended sediment load for each Mississippi River and tributary station is relatively straightforward, interpretation of the loads can be more challenging. There are several reasons for this. Heidel (1956), Walling (1977), and Van Sickle and Beschta (1983) recognized that over the course of a flood, sediment delivery from the source drainage area of a river decreases as readily erodible material is exhausted, even as water discharge (and floodplain inundation) continues to be high. Multiple floods in a single year may also carry variable suspended load, again due to variability in supply from the floodplain. This phenomenon, known as hysteresis, can result in wide variability in suspended sediment load in 
transport at a given discharge into the Mississippi River as represented in the sediment ratings curve (Mossa 1996)-depending on what stage (ascending or descending limb) of the flood it takes place. Allison et al. (2014) utilized calibrated daily turbidity measurements taken at the USGS Belle Chasse, LA, stations in WY 2008-2010 to demonstrate how the daily loads by this (measured) method differ from that derived indirectly from the sediment ratings curve. These comparisons clearly show the role of hysteresis, with (1) total suspended sediment load peaking on the rising limb of each flood, (2) variability in the peak suspended sediment load with each flood in a year (total 1-5 floods/yr post-1963 at these stations) in a way that is not directly proportional to water discharge, and (3) falling suspended sediment loads and concentrations during the water discharge peak of floods, continuing throughout the falling limb of the flood. However, when averaged over annual and longer timescales, their ratings and daily turbidity derived suspended sediment loads agreed relatively closely ( $\pm 5 \%$ to $10 \%)$.

As previous researchers have recognized (Walling 1977), the scatter in sediment ratings curves such as Figure 5 are a mixture of measurement errors and real, hysteresis-modulated variability. In addition to the curve type selected as discussed above, there are a number of measurement factors that can contribute to variability in a rating curve-derived, suspended sediment load reported for a station. They can be classified into several types, including the following:

1. Water discharge measurement errors. Water discharge, as measured by $\mathrm{ADCP}$, is shown to vary when repeat transects are conducted in rapid succession at a fixed discharge. This is due to pulsing in river velocities and compass calibration errors, among others; this variability can be reduced by averaging multiple transects (Edwards and Glysson 1999).

2. Length of record averaged. Given that annual Mississippi River floods are unique in terms of number of flood pulses/year, maximum and minimum flows, and source of the sediments in a multiple tributary system such as this, the number of years over which data are utilized to construct a sediment ratings curve will affect the resulting suspended sediment load with a given curve fit. Hence, the present study does not make use of any previously calculated sediment loads for any of the Mississippi River or tributary stations examined. All stations are also averaged for the same period of boat-based data collection (WY 1990-2013). Note that the gaps in boat-based sampling reported in Tables 1 and 2 for individual stations 
can lead to differing results between stations than if they had each been sampled an equal number of occasions at similar dates.

3. Suspended sediment sampler variability. A wide variety of samplers have been utilized to collect suspended sediment in the Mississippi River and its tributaries over the years. Different samplers are utilized at different stations, typically due to different data collection entities within and across the USGS and USACE. Even at a single station, improved technology has led to substitutions in samplers utilized through the years, often without significant co-use of both types to inter-calibrate before old technologies are retired. Different sampler intakes (isokinetic vs. non-isokinetic weighted bottles) have variable efficiencies in collecting particles of different sizes, particularly sands. Weight of sampler is also critical in that suspended particle concentrations in river channels typically increase with depth (particularly the coarser, sand fraction), and sampler weight (and hydrodynamic aspect) will affect the wire angle of the sampler deployed from the boat. Since length of wire paid out is typically the measure of apparent depth that a suspended sediment sampler is deployed, increased wire angle leads to decreased apparent vs. real depth of the sampler (Beverage 1987). Lighter samplers at a given flow velocity typically will yield a lower suspended sediment concentration. Some station operators compensate for this by utilizing a heavier sampler during the higher velocity flood periods. While this source of variability is difficult to quantify, careful documentation of the sampler type utilized at each station and how it changes through time is a first step toward determining if this is a significant factor. Tables 1 and 2 document the sampler types for each station where recorded by the operator. More details of each sampler utilized can be found on the Federal Interagency Sedimentation Project (FISP) website at http://water.usgs.gov/fisp/catalog index.html.

4. Suspended sediment sample depths. Just as sampler type is individualistic and variable through time at Mississippi River and tributary monitoring sites, so is depth at which suspended samples are collected in each vertical along the measurement transect. Shifting depths of sample intervals or even the location of verticals across the transect in a channel where suspended sediment concentrations vary laterally and with depth can impact the calculated suspended sediment load for that date and water discharge. Information about depths of sampling and location of verticals and how they vary through time at a site is even more difficult to acquire than sampler type(s) utilized. 
Table 3. Water and sediment discharge in WY1990-2013 for USGS/USACE Mississippi River monitoring stations.

\begin{tabular}{|c|c|c|c|c|c|c|c|}
\hline Station Name & $\begin{array}{l}\text { Annual } \\
\text { Water } \\
\text { Discharge in } \\
10^{11} \mathrm{ft}^{3} / \mathrm{yr}\end{array}$ & $\begin{array}{l}\text { Total } \\
\text { Suspended } \\
\text { Sediment Load } \\
\text { in } 10^{6} \text { tons/yr } \\
\text { (quadratic) }\end{array}$ & \begin{tabular}{|l|} 
Total \\
Suspended \\
Sediment Load \\
in $10^{6}$ tons/yr \\
(best fit)
\end{tabular} & $\begin{array}{l}\text { Maximum Annual } \\
\text { Total Suspended } \\
\text { Load in } 10^{6} \\
\text { tons/yr } \\
\text { (year) }\end{array}$ & $\begin{array}{l}\text { Minimum Annual } \\
\text { Total Suspended } \\
\text { Load } \\
\text { in } 10^{6} \text { tons/yr } \\
\text { (year) }\end{array}$ & $\begin{array}{l}\text { Suspended } \\
\text { Sand Load } \\
\text { in } 10^{6} \text { tons/yr } \\
\text { (quadratic) }\end{array}$ & $\begin{array}{l}\text { Suspended } \\
\text { Sand Load } \\
\text { in } 10^{6} \text { tons/yr } \\
\text { (best fit) }\end{array}$ \\
\hline $\begin{array}{l}\text { Grafton/ } \\
\text { Below Grafton, IL }\end{array}$ & 41.9 & 23.8 & 20.5 & 43.8 (1993) & $5.8(2012)$ & 2.8 & 2.5 \\
\hline St. Louis, MO & 71.2 & 78.8 & $78.8(q)$ & $183.4(1993)$ & $17.8(2006)$ & 13.7 & $13.7(q)$ \\
\hline Chester, IL & 74.3 & 69.3 & $69.3(q)$ & 174.3 (1993) & 21.5 (2006) & 16.8 & $16.8(q)$ \\
\hline Thebes, IL & 77.3 & 94.0 & 88.7 & $201.2(1993)$ & $23.0(2006)$ & 15.6 & $15.6(q)$ \\
\hline Arkansas City, AR & 198.8 & 74.9 & $74.9(q)$ & $112.0(1993)$ & $36.3(2000)$ & 13.6 & $13.6(q)$ \\
\hline Vicksburg, MS & 209.2 & 77.3 & 77.2 & $111.8(1993)$ & $40.1(2006)$ & 15.8 & 16.0 \\
\hline Natchez, MS & 210.4 & 75.6 & 77.1 & $111.9(2010)$ & 40.7 (2006) & 14.9 & 14.8 \\
\hline Tarbert Landing, MS & 169.1 & undetermined & undetermined & undetermined & undetermined & undetermined & undetermined \\
\hline Old River Outfall, LA & 50.1 & undetermined & undetermined & undetermined & undetermined & undetermined & undetermined \\
\hline Simmesport, LA & 72.5 & undetermined & undetermined & undetermined & undetermined & undetermined & undetermined \\
\hline Baton Rouge, LA & 162.9 * & 82.0 & 82.6 & $119.6(2010)$ * & $36.0(2006)$ * & 21.0 & 21.8 \\
\hline Belle Chasse, LA & 163.8 & 81.6 & $81.6(q)$ & $129.4(1993)$ & $22.5(2006)$ & 14.2 & $14.2(q)$ \\
\hline
\end{tabular}

*Incomplete water discharge data available for WY1996-1997 
Table 4. Water and sediment discharge in WY1990-2013 for USGS/USACE monitoring stations in Mississippi River tributaries.

\begin{tabular}{|c|c|c|c|c|c|c|c|}
\hline Station Name & $\begin{array}{l}\text { Mean } \\
\text { Annual } \\
\text { Water } \\
\text { Discharge in } \\
10^{11} \mathrm{ft}^{3} / \mathrm{yr}\end{array}$ & $\begin{array}{l}\text { Total } \\
\text { Suspended } \\
\text { Sediment Load } \\
\text { in } 10^{6} \text { tons/yr } \\
\text { (quadratic) }\end{array}$ & $\begin{array}{l}\text { Total } \\
\text { Suspended } \\
\text { Sediment Load } \\
\text { in } 10^{6} \text { tons/yr } \\
\text { (best fit) }\end{array}$ & $\begin{array}{l}\text { Maximum } \\
\text { Annual Total } \\
\text { Suspended } \\
\text { Load } \\
\text { in } 10^{6} \text { tons/yr } \\
\text { (year) }\end{array}$ & $\begin{array}{l}\text { Minimum } \\
\text { Annual Total } \\
\text { Suspended } \\
\text { Load } \\
\text { in } 10^{6} \text { tons/yr } \\
\text { (year) }\end{array}$ & $\begin{array}{l}\text { Suspended } \\
\text { Sand Load } \\
\text { in } 10^{6} \text { tons/yr } \\
\text { (quadratic) }\end{array}$ & $\begin{array}{l}\text { Suspended } \\
\text { Sand Load } \\
\text { in } 10^{6} \text { tons/yr } \\
\text { (best fit) }\end{array}$ \\
\hline Missouri (Yankton, SD) & 6.3 & 0.16 & 0.21 & $0.52(1997)$ & $0.02(2008)$ & 0.009 & 0.009 (q) \\
\hline Missouri (Sioux City, IA) & 9.8 & Limited data & Limited data & Limited data & Limited data & Limited data & Limited data \\
\hline Missouri (Omaha, NE) & 11.3 & Limited data & Limited data & Limited data & Limited data & Limited data & Limited data \\
\hline Missouri (Nebraska City, NE) & 13.3 & Limited data & Limited data & Limited data & Limited data & Limited data & Limited data \\
\hline Missouri (St. Joseph, MO) & 15.4 & 57.0 & 48.8 & 211.6 (2011) & $8.6(2006)$ & 12.3 & $12.3(q)$ \\
\hline Missouri (Kansas City, MO) & 18.2 & 73.6 & 61.0 & 193.2 (2011) & $10.2(2002)$ & 14.1 & 12.6 \\
\hline Missouri (Hermann, MO) & 29.5 & 78.7 & 75.0 & $195.3(1993)$ & $12.5(2006)$ & 18.5 & 19.0 \\
\hline Illinois (Valley City, IL) & 8.3 & 7.8 & 7.4 & 14.8 (1993) & $2.1(2012)$ & 0.42 & 0.13 \\
\hline Meramec (Eureka, MO) & 1.2 & 0.75 & $0.75(q)$ & 1.3 (1993) & $0.11(2000)$ & 0.13 & $0.13(q)$ \\
\hline Kaskaskia (Venedy Station, IL) & 1.3 & 1.5 & $1.5(q)$ & $2.5(2013)$ & $0.43(1992)$ & 0.20 & $0.20(q)$ \\
\hline Big Muddy (Murfreesboro, IL) & 0.70 & 0.07 & 0.07 & $0.08(2010)$ & $0.06(2008)$ & 0.003 & $0.003(q)$ \\
\hline Ohio (Dam 53 near Grand Chain, IL) & 94.7 & 32.8 & $32.8(q)$ & $46.5(1997)$ & $13.8(2000)$ & 3.0 & $3.0(q)$ \\
\hline Obion (Obion, TN) & $0.89 *$ & 0.58 & 0.62 & $0.85(2003)$ * & $0.45(2012)$ * & 0.05 & $0.05(q)$ \\
\hline Hatchie (Rialto, TN) & $0.98+$ & 0.15 & 0.16 & $0.21(2004) \dagger$ & $0.11(2007) \dagger$ & 0.04 & $0.04(q)$ \\
\hline Loosahatchie (Arlington, TN) & 0.13 & 0.57 & $0.57(q)$ & $1.3(2002)$ & $0.10(2007)$ & Limited data & Limited data \\
\hline Wolf (Germantown, TN) & $0.31+$ & 0.27 & 0.36 & $1.2(2011)$ & $0.09(2007)$ & 0.08 & $0.08(q)$ \\
\hline St. Francis (Parkin/Madison, AR) & $0.65^{\wedge}$ & 0.40 & $0.40(q)$ & $0.60(1991)^{\wedge}$ & $0.24(2001)$ & 0.02 & $0.02(q)$ \\
\hline White (Devall's Bluff, AR) & 8.6 & 2.7 & 2.8 & $4.9(2010)$ & $0.87(2006)$ & 1.1 & 1.2 \\
\hline Arkansas (Little Rock, AR) & 15.5 & 5.1 & $5.1(q)$ & $16.1(1993)$ & $0.9(2006)$ & 2.3 & $2.3(q)$ \\
\hline Yazoo (Long Lake, MS) & $5.5 * *$ & 5.6 & $5.6(q)$ & $9.1(2002) * *$ & $2.7(2012) * *$ & 0.27 & 0.28 \\
\hline
\end{tabular}




\begin{tabular}{|c|c|c|c|c|c|c|c|}
\hline Station Name & $\begin{array}{l}\text { Mean } \\
\text { Annual } \\
\text { Water } \\
\text { Discharge in } \\
10^{11} \mathrm{ft}^{3} / \mathrm{yr}\end{array}$ & $\begin{array}{l}\text { Total } \\
\text { Suspended } \\
\text { Sediment Load } \\
\text { in } 10^{6} \text { tons/yr } \\
\text { (quadratic) }\end{array}$ & $\begin{array}{l}\text { Total } \\
\text { Suspended } \\
\text { Sediment Load } \\
\text { in } 10^{6} \text { tons/yr } \\
\text { (best fit) }\end{array}$ & $\begin{array}{l}\text { Maximum } \\
\text { Annual Total } \\
\text { Suspended } \\
\text { Load } \\
\text { in } 10^{6} \text { tons/yr } \\
\text { (year) }\end{array}$ & $\begin{array}{l}\text { Minimum } \\
\text { Annual Total } \\
\text { Suspended } \\
\text { Load } \\
\text { in } 10^{6} \text { tons/yr } \\
\text { (year) }\end{array}$ & $\begin{array}{l}\text { Suspended } \\
\text { Sand Load } \\
\text { in } 10^{6} \text { tons/yr } \\
\text { (quadratic) }\end{array}$ & $\begin{array}{l}\text { Suspended } \\
\text { Sand Load } \\
\text { in } 10^{6} \text { tons/yr } \\
\text { (best fit) }\end{array}$ \\
\hline Big Black (Bovina, MS) & 1.3 & 0.70 & $0.70(q)$ & $1.1(2003)$ & $0.28(2000)$ & 0.40 & 0.11 \\
\hline Homochitto (Rosetta, MS) & 0.35 & 0.49 & $0.49(q)$ & $1.2(1994)$ & $0.03(2000)$ & 0.31 & $0.31(q)$ \\
\hline
\end{tabular}

*Incomplete water discharge data available for WY1996-2001

† Incomplete water discharge data available for WY1990-2003

+ Incomplete water discharge data available for WY1990

^Water discharge data from Parkin, total sediment from Parkin and Madison combined, incomplete water discharge data available for WY1995-1997, 2003-2004, 2011-2013

** Incomplete water discharge data available for WY1990-1995 


\section{Discussion of Results}

In this section, the suspended sediment loads for the Mississippi River stations and each tributary are presented, first for the WY 1990-2013 period compiled by the present study and then in comparison to the WY 1970-1978 records compiled by Keown et al. (1981). Detailed assessments and statistical analyses of trends are not reported in the present study, nor are the trends attributed to specific natural or anthropogenic factors such as changes in the hydrologic regime and/or basin sediment supply or construction of dikes, revetments, cutoffs, levees, dams, or diversions. These and other comprehensive analyses are planned for future MRG\&P efforts. Following these sections discussing calculated water and sediment discharge results, a set of recommendations about station location and sampling methodology will be outlined based on the analysis of the limitations observed in the data utilized by the present study.

\subsection{Water and suspended sediment loads for WY 1990-2013 for the Mississippi River and major tributaries}

Mean annual water discharge and total and sand-fraction suspended sediment loads are reported for Mississippi River stations in Table 3, and for 17 major tributaries (16 measured and the Upper Mississippi River determined by subtraction) in Table 4. Figure 6 and Figures 8-9 show this information in diagrammatic form to present the points of tributary input relative to the location of Mississippi River stations.

\subsubsection{Water discharge}

The calculation of daily water discharge is a necessary first step in converting the sediment ratings curves to daily suspended sediment loads. However, tallying annual water discharge for stations and examining the water budget moving from upstream to downstream is also a means of assessing whether (1) there are any measurement or calculation errors in water discharge by the operator that may impact sediment budgeting, (2) the selected tributary inputs are responsible for the vast majority of water and sediment inputs to stations downstream, and (3) there is any net loss/gain in water that may come from other sources such as groundwater exchange that may impact stream power and sediment transport capacity. 
Annual mean water discharge for the seven stations (Figure 6) examined downstream of the last dam on the Missouri River (e.g., Gavins Point Dam) immediately upstream of the Yankton, SD, station increase by $368 \%$ (6.3 to $29.5 \times 10^{11}$ cubic feet per year [ $\left.\mathrm{ft} 3 / \mathrm{yr}\right]$ ). This indicates that the bulk of the water reaching the confluence below the Hermann, MO, station is derived from the tributaries entering the Missouri downstream of the dams (e.g., Platte, Kansas, Osage). The suspended sediment load of the Missouri at the confluence will be strongly controlled by the delivery from these tributaries, as well as the trapping efficiency of the Upper Missouri dams, as will be discussed later in this report.

Figure 6. Annual average water discharge (in $10^{11} \mathrm{ft}^{3} / \mathrm{yr}$ ) for Mississippi River stations and Missouri River stations (red circles) and tributary inputs in the reach from St. Louis, MO, to Louisiana integrated for the period of WY 1990-2013.

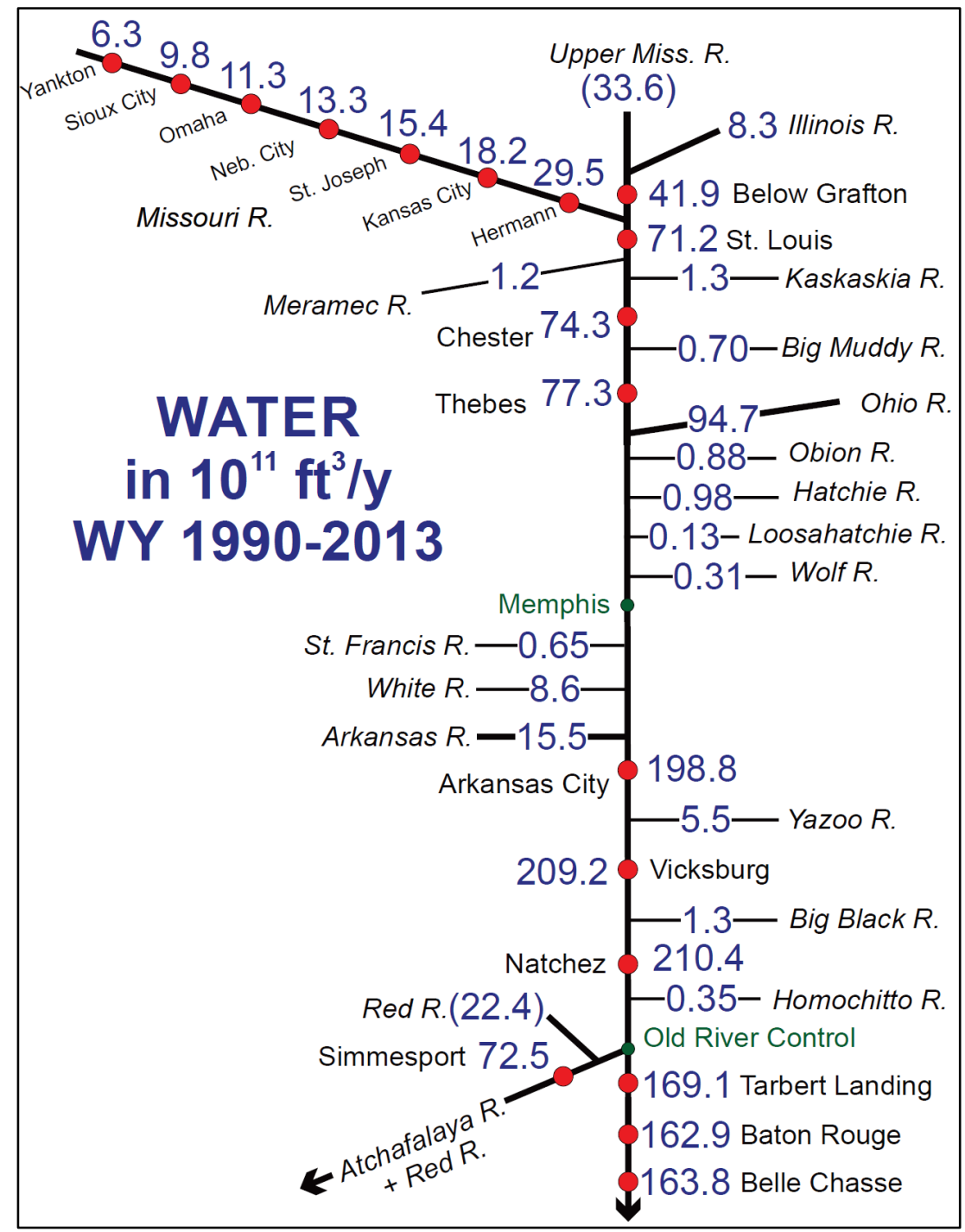


A water budget from the confluence station (St. Louis, MO) to Belle Chasse, LA, is constructed in Table 5 by taking calculated annual mean water discharges from WY 1990-2013 (Table 3) and examining how the volume of water changes at the next Mississippi River station, accounting for the calculated tributary water inputs (Table 4). Through the entire reach, budgeting accounts for all but $<0.1 \%$ to $5.3 \%$ of the measured annual water flow at stations. This suggests that the calculated water discharges by the operator(s) are accurate within a few percent and that the selected tributaries initially suggested by Keown et al. (1981) account for most of water (and sediment) entering the main stem below St. Louis, MO. An example of this accuracy is that there is only a $0.2 \%$ loss between Thebes, IL, and Arkansas City, AR, despite the measurement errors associated with calculating eight tributary flows that are added to the Mississippi River's water discharge in this reach (Table 5). The largest unaccounted-for water fraction occurs at Tarbert Landing, MS, and Baton Rouge, LA. In part, this may be a function of complexities associated with measuring the outflows through three channels at Old River Control (e.g., Hydropower, Low Sill, Auxiliary) and their impact on the annual summing conducted to yield these averages. Allison et al. (2012) also suggest the possibility of groundwater loss in the Tarbert Landing, MS, to Belle Chasse, LA, reach as an additional mechanism.

Table 5. Water budget of Mississippi River from St. Louis, MO, to Belle Chasse, LA, for average annual flow from WY 1990-2013.

\begin{tabular}{|l|c|c|c|}
\hline STATIONS & $\begin{array}{c}\text { ANNUAL WATER } \\
\text { DISCHARGE } \\
\left.\text { (in 1011 } \mathrm{ft}^{3} / \mathrm{yr}\right)\end{array}$ & $\begin{array}{c}\text { TRIBUTARY } \\
\text { ADDITION } \\
\left.\text { (in 1011 } \mathrm{ft}^{3} / \mathrm{yr}\right)\end{array}$ & $\begin{array}{c}\% \text { loss/gain } \\
\left(10^{11} \mathrm{ft}^{3} / \mathrm{yr}\right)\end{array}$ \\
\hline Illinois+Upper Miss+Missouri & & 71.4 & \\
\hline ST LOUIS, MO & 71.2 & & $-0.3(-0.2)$ \\
\hline Meramec+Kaskaskia & 74.3 & & $0.8(0.6)$ \\
\hline CHESTER, IL & & 0.7 & \\
\hline Big Muddy & 77.3 & & $3.1(2.3)$ \\
\hline THEBES, IL & & 121.8 & \\
\hline $\begin{array}{l}\text { Ohio+Obion+Hatchie+Lossahatchie+Wolf+St. } \\
\text { Francis+White+Arkansas }\end{array}$ & 198.8 & & $-0.2(-0.3)$ \\
\hline ARKANSAS CITY, AR & & 5.5 & \\
\hline Yazoo & 209.2 & & $2.4(4.9)$ \\
\hline VICKSBURG, MS & & 1.3 & \\
\hline Big Black & 210.4 & & $-0.05(-0.1)$ \\
\hline NATCHEZ, MS & & & \\
\hline
\end{tabular}




\begin{tabular}{|l|c|c|c|}
\hline STATIONS & $\begin{array}{c}\text { ANNUAL WATER } \\
\text { DISCHARGE } \\
\left.\text { (in } 10^{11} \mathrm{ft}^{3} / \mathrm{yr}\right)\end{array}$ & $\begin{array}{c}\text { TRIBUTARY } \\
\text { ADDITION } \\
\left.\text { (in } 10^{11} \mathrm{ft}^{3} / \mathrm{yr}\right)\end{array}$ & $\begin{array}{c}\% \text { loss/gain } \\
\left(10^{11} \mathrm{ft}^{3} / \mathrm{yr}\right)\end{array}$ \\
\hline Homochitto+(-) Old River Control & & -49.8 & \\
\hline TARBERT LANDING, MS & 169.1 & & $5.3(8.5)$ \\
\hline BATON ROUGE, LA & 162.9 & & $-3.8(-6.2)$ \\
\hline BELLE CHASSE, LA & 163.8 & & $0.5(0.9)$ \\
\hline
\end{tabular}

Large floods have a significant influence on the annual water discharge averages obtained for the $24 \mathrm{yr}$ study period (WY 1990-2013). Basin-wide, large floods in terms of peak water discharge occurred in 1993, 1997, 2008, and 2011. The influence of these floods on the Mississippi River and tributary stations examined in the present study is variable, depending on where the bulk of precipitation fell. Table 1 and Figure 7 show that the 1993 flood was the largest in the reach from Thebes, IL, to the Missouri confluence, while from Arkansas City, AR, to Baton Rouge, LA, it was exceeded by the 2011 flood. Although the 1993 flood is not the highest in terms of peak flow in the lower Mississippi River, it was a year of sustained high flows capable of performing a significant amount of geomorphic work and sediment transport (Horowitz 2010). Some years also have large integrated annual water discharge at Mississippi River stations that did not experience major peak flood discharges-2010 is an example where five individual moderate flood peaks together produced a larger flood year than 2011 at some stations (e.g., Baton Rouge and Belle Chasse, LA; Natchez, MS). The station at Belle Chasse, LA, is impacted by the release of water in large floods at the Bonnet Carre Spillway when water discharges exceed 1.25 million cubic feet per second ( $\mathrm{ft} 3 / \mathrm{sec}$ ) at Tarbert Landing, MS; hence, all the years where the structure was operated (i.e, 1993, 1997, 2008, and 2011) have similar peak flood discharges (Figure 7). Both Belle Chasse and Baton Rouge, LA, stations are impacted by a second flood control structure (Morganza Spillway) that is operated when discharge at Tarbert Landing, MS, exceeds 1.5 million $\mathrm{ft} 3 / \mathrm{sec}$. The structure was operated in 2011, and that outflow volume is not accounted for in the Baton Rouge and Belle Chasse, LA, water volume loss relative to Tarbert Landing, MS, shown in Table 5. 
Figure 7. Annual water discharge for the three largest flow years in the WY 1990-2013 period for Mississippi River stations at Thebes, IL; Natchez, MS; and Belle Chasse, LA. The "Tarbert Spillway Opening Discharge" line in the Belle Chasse plot refers to the discharge (at Tarbert Landing, MS) that triggers opening of the Bonnet Carre Spillway between Baton Rouge and Belle Chasse, LA, stations. This limits flow at Belle Chasse, LA, to a maximum of 1.25 million $\mathrm{ft}^{3} / \mathrm{sec}$.

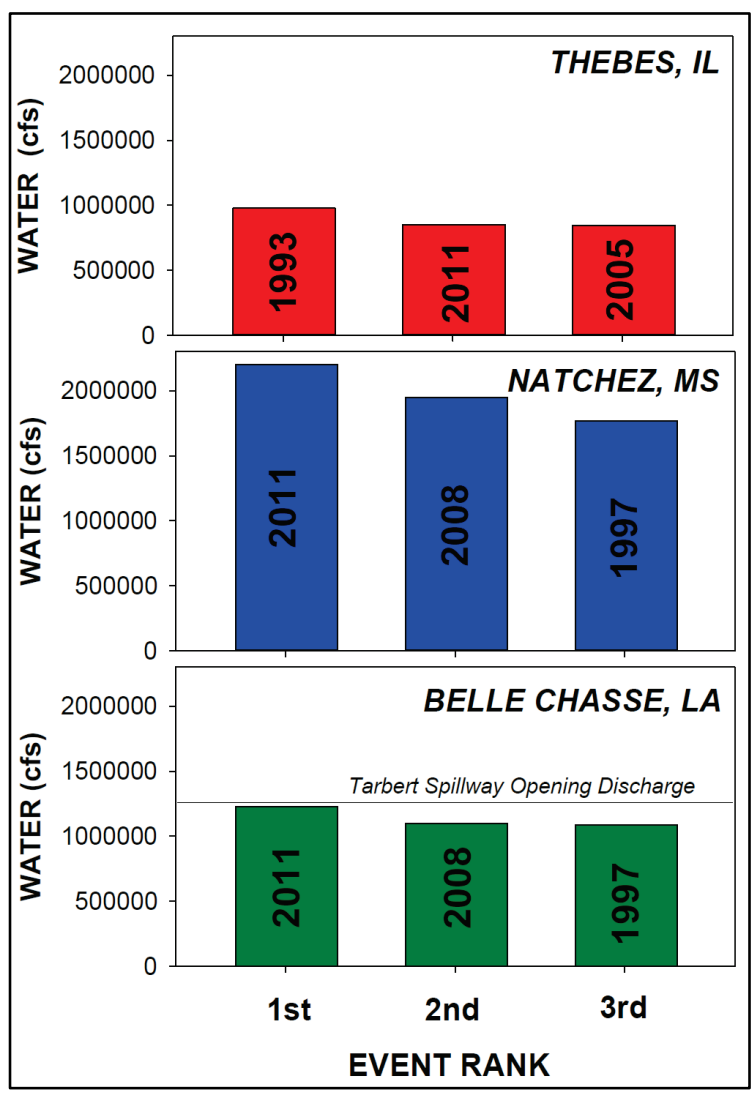

\subsubsection{Total and sand-sized suspended sediment discharge.}

Following the approach for the water budget, a sediment budget, tracking downstream changes in Mississippi River main-stem total and sand suspended loads (in tons of change per year) corrected for tributary inputs is presented in Tables 6 and 7 and in graphical form in Figures 8 and 9. With respect to total sediment load (Table 6), changes between stations range from $1.2 \%$ to $75.6 \%$. Partly this can be anticipated as a function of compounded errors with the water discharge and the hysteresis- and sampling-induced scatter in the ratings curve outlined in Section 3.4. However, several other factors may be present in the Mississippi River stations, some of which may suggest these budgetary changes cannot be 
automatically ascribed to real changes in load. The stations at St. Louis, MO, and Chester, IL, have the fewest $(<50)$ measurements of any of the stations studied and they were only collected over a limited number of water years (2010-2014). The absence of large events such as 1993, 1998, and 2008 in the sample record would tend to lessen the ratings curve total load, and these stations are more than 10 million tons/yr below the next station downstream (Thebes, IL) that was well-sampled throughout the period studied. The Thebes, IL, record is also only $2.6 \%$ less than the combined measured tributary inputs of the Illinois, Upper Mississippi, and Missouri Rivers (Table 6), lending further credence to the conclusion that much of this difference is an artifact of sampling density.

Table 6. Total suspended sediment budget of Mississippi River from St. Louis, MO, to Belle Chasse, LA, for average annual flow from WY 1990-2013.

\begin{tabular}{|c|c|c|c|}
\hline STATIONS & $\begin{array}{l}\text { ANNUAL TOTAL } \\
\text { SUSPENDED } \\
\text { DISCHARGE } \\
\text { (in } 10^{6} \text { tons/yr) }\end{array}$ & $\begin{array}{c}\text { TRIBUTARY } \\
\text { ADDITION } \\
\text { (in } 10^{6} \text { tons/yr) }\end{array}$ & $\begin{array}{l}\% \text { loss/gain } \\
\left(10^{6} \text { tons/yr) }\right.\end{array}$ \\
\hline Illinois+Upper Miss+Missouri & & 95.5 & \\
\hline ST LOUIS, MO & 78.8 & & $-21.2(-16.7)$ \\
\hline Meramec+Kaskaskia & & 2.3 & \\
\hline CHESTER, IL & 69.4 & & $-16.9(-11.7)$ \\
\hline Big Muddy & & 0.1 & \\
\hline THEBES, IL & 88.7 & & $27.6(19.2)$ \\
\hline $\begin{array}{l}\text { Ohio+Obion+Hatchie+Lossahatchie } \\
+ \text { Wolf+St. Francis+White+Arkansas }\end{array}$ & & 42.8 & \\
\hline ARKANSAS CITY, AR & 74.9 & & $-75.6(-56.6)$ \\
\hline Yazoo & & 5.6 & \\
\hline VICKSBURG, MS & 77.3 & & $-4.3(-3.2)$ \\
\hline Big Black & & 0.7 & \\
\hline NATCHEZ, MS & 77.1 & & $-3.8(-2.9)$ \\
\hline Homochitto+(-) Old River Control & & $0.5-?$ & \\
\hline TARBERT LANDING, MS & Not available & & $?(?)$ \\
\hline BATON ROUGE, LA & 82.6 & & $?(?)$ \\
\hline BELLE CHASSE, LA & 81.6 & & $-1.2(1.0)$ \\
\hline
\end{tabular}


Table 7. Suspended sand budget of Mississippi River from St. Louis, MO, to Belle Chasse, LA, for average annual flow from WY 1990-2013.

\begin{tabular}{|c|c|c|c|}
\hline STATIONS & $\begin{array}{c}\text { ANNUAL SUSPENDED } \\
\text { SAND } \\
\text { DISCHARGE } \\
\text { (in } 10^{6} \text { tons } / \mathrm{yr} \text { ) }\end{array}$ & $\begin{array}{c}\text { TRIBUTARY } \\
\text { ADDITION } \\
\text { (in } 10^{6} \text { tons/yr) }\end{array}$ & $\begin{array}{c}\% \text { loss/gain } \\
\text { (106 tons/yr) }\end{array}$ \\
\hline Illinoi+Upper Miss+Missouri & & 21.5 & \\
\hline ST LOUIS, MO & 13.7 & & $-56.9(-7.8)$ \\
\hline Meramec+Kaskaskia & & 0.3 & \\
\hline CHESTER, IL & 16.8 & & $20.0(3.2)$ \\
\hline Big Muddy & & $<0.1$ & \\
\hline THEBES, IL & 15.6 & & $-7.7(-1.2)$ \\
\hline $\begin{array}{l}\text { Ohio+Obion+Hatchie+Lossahatchie+Wolf+ } \\
\text { St. Francis+White+Arkansas }\end{array}$ & & 3.7 & \\
\hline ARKANSAS CITY, AR & 13.6 & & $-41.9(-5.7)$ \\
\hline Yazoo & & 0.3 & \\
\hline VICKSBURG, MS & 16.0 & & $15.1(2.1)$ \\
\hline Big Black & & 0.1 & \\
\hline NATCHEZ, MS & 14.8 & & $-8.8(-1.3)$ \\
\hline Homochitto+(-) Old River Control & & $0.3-?$ & \\
\hline TARBERT LANDING, MS & Not available & & $?(?)$ \\
\hline BATON ROUGE, LA & 21.8 & & $?(?)$ \\
\hline BELLE CHASSE, LA & 14.2 & & $53.5(7.6)$ \\
\hline
\end{tabular}

Farther downstream, there is a $76 \%$ drop in apparent total suspended sediment load in the next station (Arkansas City, AR) and then differences in load downstream between the other two stations above Old River Control (i.e., Vicksburg and Natchez, MS) are small ( 4\%). All three stations are operated by the USACE Vicksburg District. Two factors may complicate the causality of the data at these three Mississippi River stations. There is a significant time gap through most of the $1990 \mathrm{~s}$ (including large floods) that was a result of the present study excluding this data because of indications that there were calculation errors in the dataset. This included multiple samples per WY where sand load exceeded fine load. 
Figure 8. Annual average total suspended loads (computed in $10^{6}$ tons/yr) for Mississippi River stations and Missouri River stations (red circles) and tributary inputs in the reach from St. Louis, MO, to Louisiana integrated for the period of WY 1990-2013. Asterisks in the Missouri River refer to insufficient sediment data to calculate a load and in the Red River, Simmesport, LA, and Tarbert Landing, MS, stations refer to incomplete data analysis by the station operator(s). Asterisks at St. Louis, MO, and Chester, MO, refer to the interpretation that the data are over such a limited time frame (WY 20102014) that it impacts the load calculated.

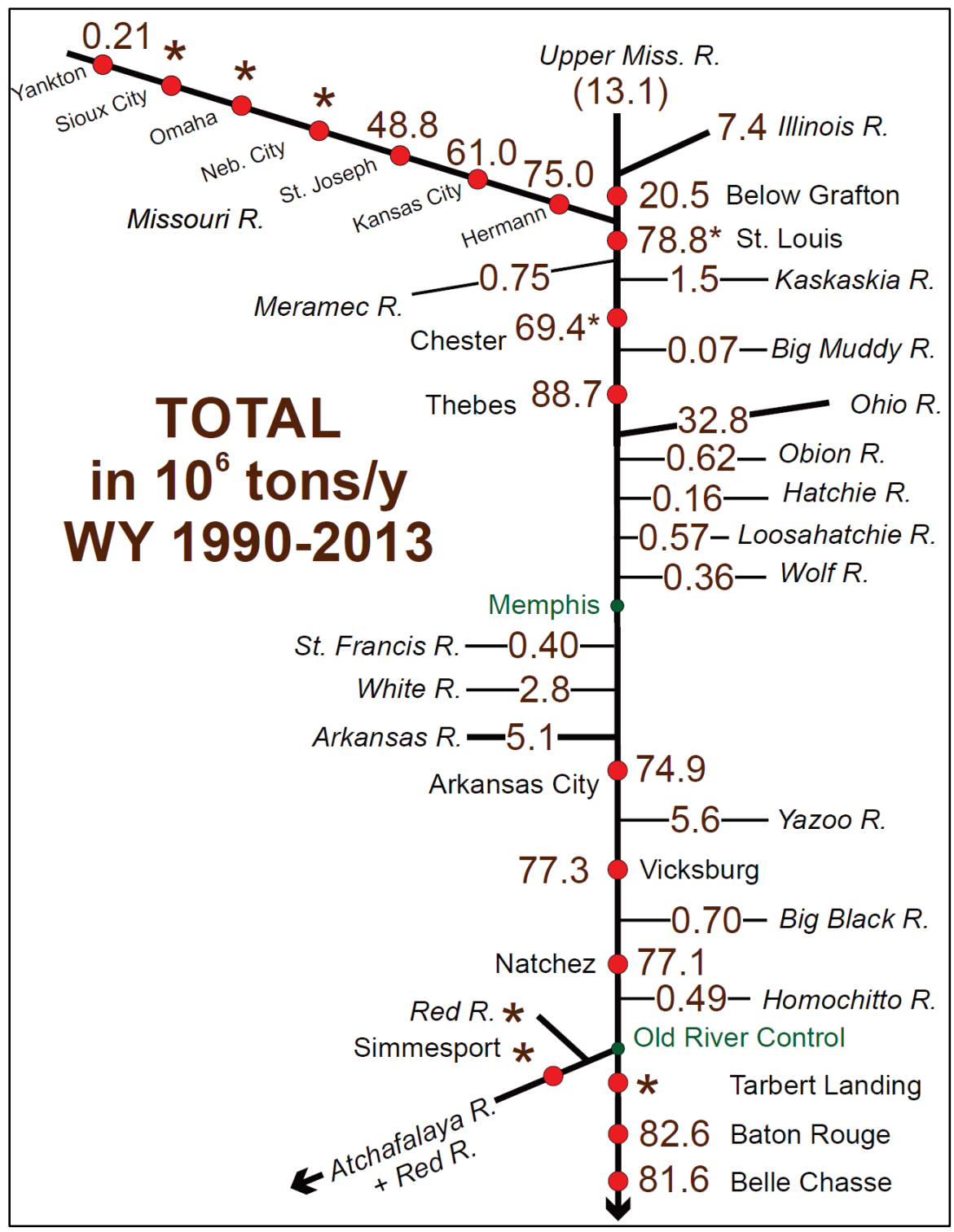


Figure 9. Annual average total suspended sand load (computed in $10^{6}$ tons/yr) for Mississippi River stations and Missouri River stations (red circles) and tributary inputs in the reach from St. Louis, MO, to Louisiana integrated for the period of WY 1990-2013. Asterisks in the Missouri River and Kaskaskia River refer to insufficient sediment data to calculate a load and in the Red River, Simmesport, LA, and Tarbert Landing, MS, stations refer to incomplete data analysis by the station operator(s). Asterisks at St. Louis, MO, and Chester, MO, refer to the interpretation that the data are over such a limited time frame (WY 2010-2014) that it impacts the load calculated.

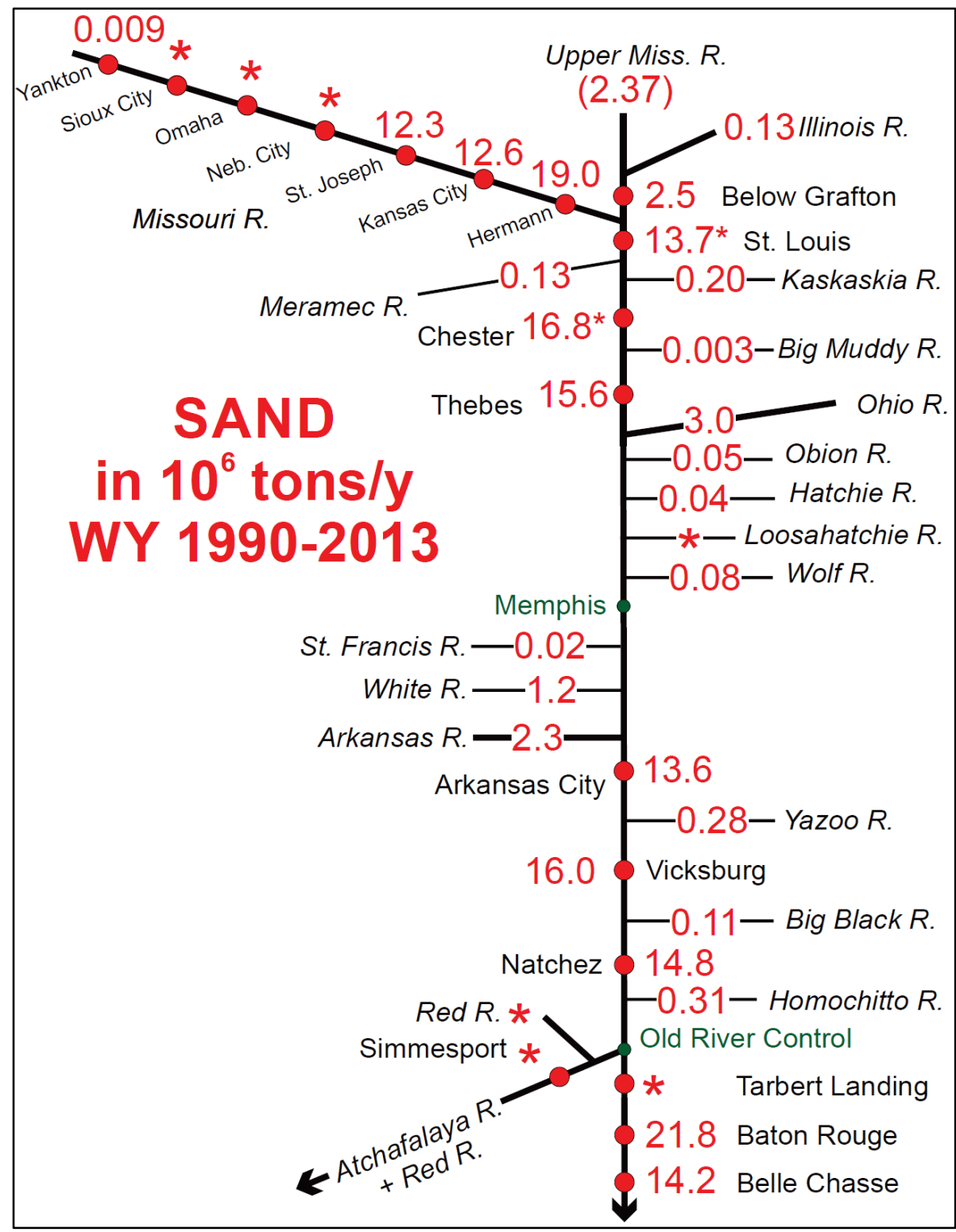

There were also questions if laboratory and data entry procedures were followed. A second factor is related to the sampler applied at these three stations. The reach where these stations are located, between the Ohio River confluence and Old River Control, is the reach where water discharges are highest, with flows above 2.2 million $\mathrm{ft} 3 / \mathrm{sec}$ measured in 
2011 (Table 1). Stations below Old River Control (e.g., Baton Rouge and Belle Chasse, LA), in spite of lower flows due to the loss of water to the Atchafalaya River distributary, utilize the 285 lb D-99 sampler in higher water discharges to minimize wire-angle induced shallowing of the fish above the stated depth. The USACE Vicksburg District stations utilize the $105 \mathrm{lb}$ P-61 sampler even at high flow velocities (i.e., high discharge). Given that the Mississippi River tends to have increasing sediment concentrations with depth with sand-sized material (Ramirez and Allison 2013), this would result in a reduced concentration in the sampler and a calculated lower sand suspended load, which would impact the total load (which includes sand) as well. Fines tend to be relatively homogenized with depth during high flow events (Ramirez and Allison 2013). The USGS has collected a relatively small number of samples (28) in WY 1990-1994 and 2008 using the D96/99 methods applied at Baton Rouge and Belle Chasse, LA, at a station approximately 1 river mile south of the USACE Vicksburg, MS, station. Utilizing the USACE water discharges to limit that source of variability (USGS also made water discharge measurements) and quadratic best fit for both sediment ratings curves (Figure 10), the USGS calculated average total suspended load was $94.8 \pm 51.2$ million tons/yr, and suspended sand was $31.5 \pm 11.6$ million tons/yr. This is 17.5 million tons/yr (23\%) higher in total load, almost all of which can be attributed to the difference in sand load (15.7 million tons, $99 \%$ higher than the USACE values). While these differences are not statistically significant, the preponderance of the increase concentrated in the sand load supports the possible link to the lighter sampler, wire angle effect.

Other sources of variability in the total and sand-suspended load calculated for stations may also be affecting the values in Tables 6 and 7. As mentioned previously, large floods have a disproportionate effect on annual sediment loads. The three largest floods in terms of total suspended load are presented in Figure 11 for three stations between St. Louis, MO, and the Gulf of Mexico. Figure 12 presents all the suspended loads in the WY 19902013 period at the same stations for comparison. Often, these large floods are not sampled in the semi-regular sampling at stations occupied by the USGS and USACE. Table 1 shows that the seven Mississippi River stations above Old River Control have sampled at a maximum of $55 \%$ to $82 \%$ of the maximum flow observed in WY 1990-2013. This limited number of data points at the upper water discharge end of the sediment ratings can result in large errors in calculated flux. An example can be seen in Figure 10 where the USGS sampling specific to the large 2008 flood defined a clear upper 
limit to the ratings curve, where the semi-regular USACE pattern, which was not designed to capture large flow events, had limited data in the higher part of the hydrograph, which increases uncertainties. The two stations below Old River Control have a much better record of sampling large events (Baton Rouge, LA, 100\%; Belle Chasse, LA, 93\%) because of the coordinated effort between USGS and USACE New Orleans District to track large floods for operation of the flood control structures (e.g., Old River Control, Morganza, and Bonnet Carre Spillways).

Figure 10. Sediment ratings curve for the Vicksburg, MS, stations on the Mississippi River operated by the USACE Vicksburg District and USGS showing the higher apparent sand (lower plot) attributed to the heavier D96/D99 sampler utilized. quadratic best fit equations utilized for both data sets.

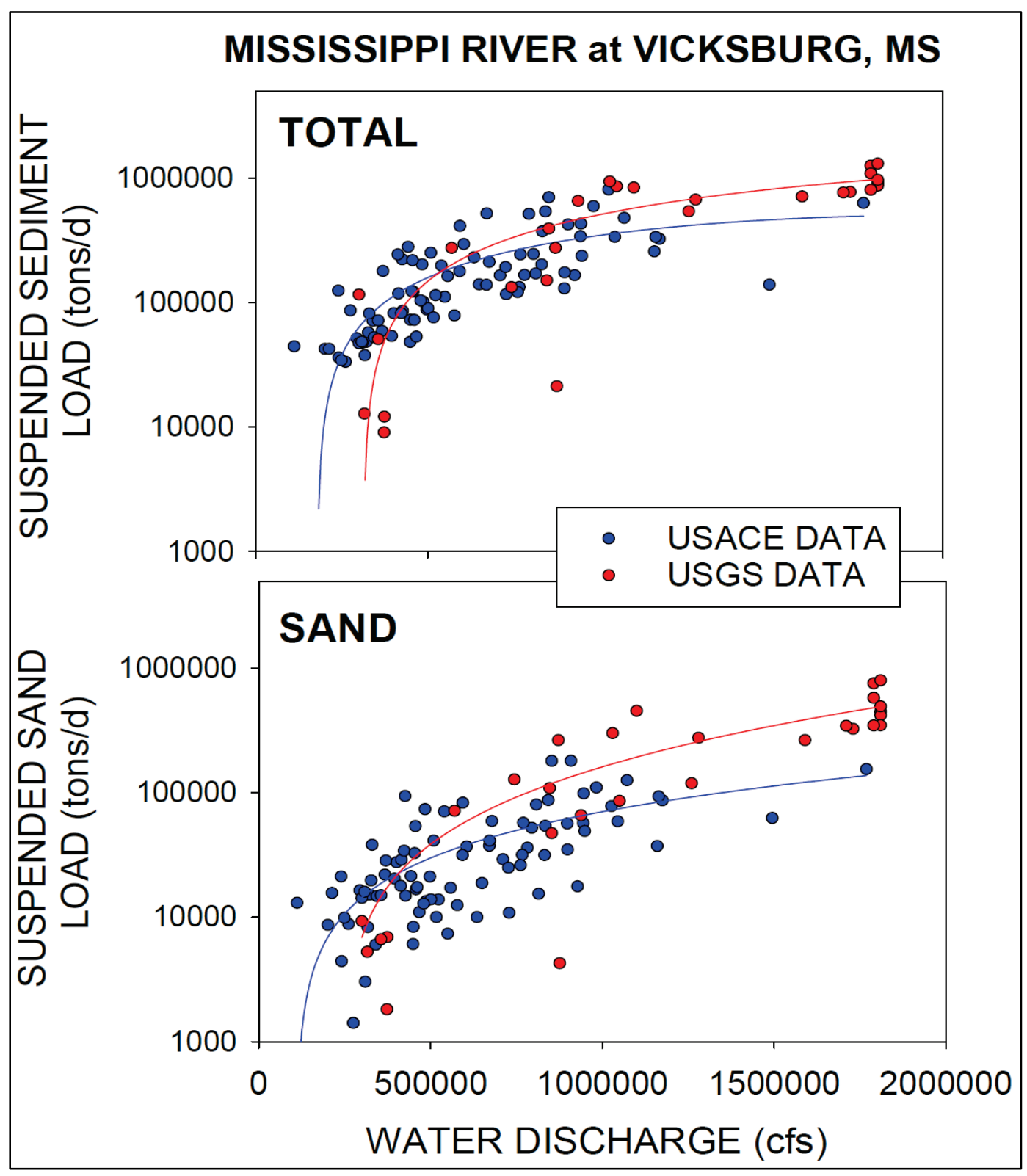


Figure 11. Annual total suspended sediment discharge for the three largest sediment years in the WY 1990-2013 period for Mississippi River stations at Thebes, IL; Natchez, MS; and Belle Chasse, LA.

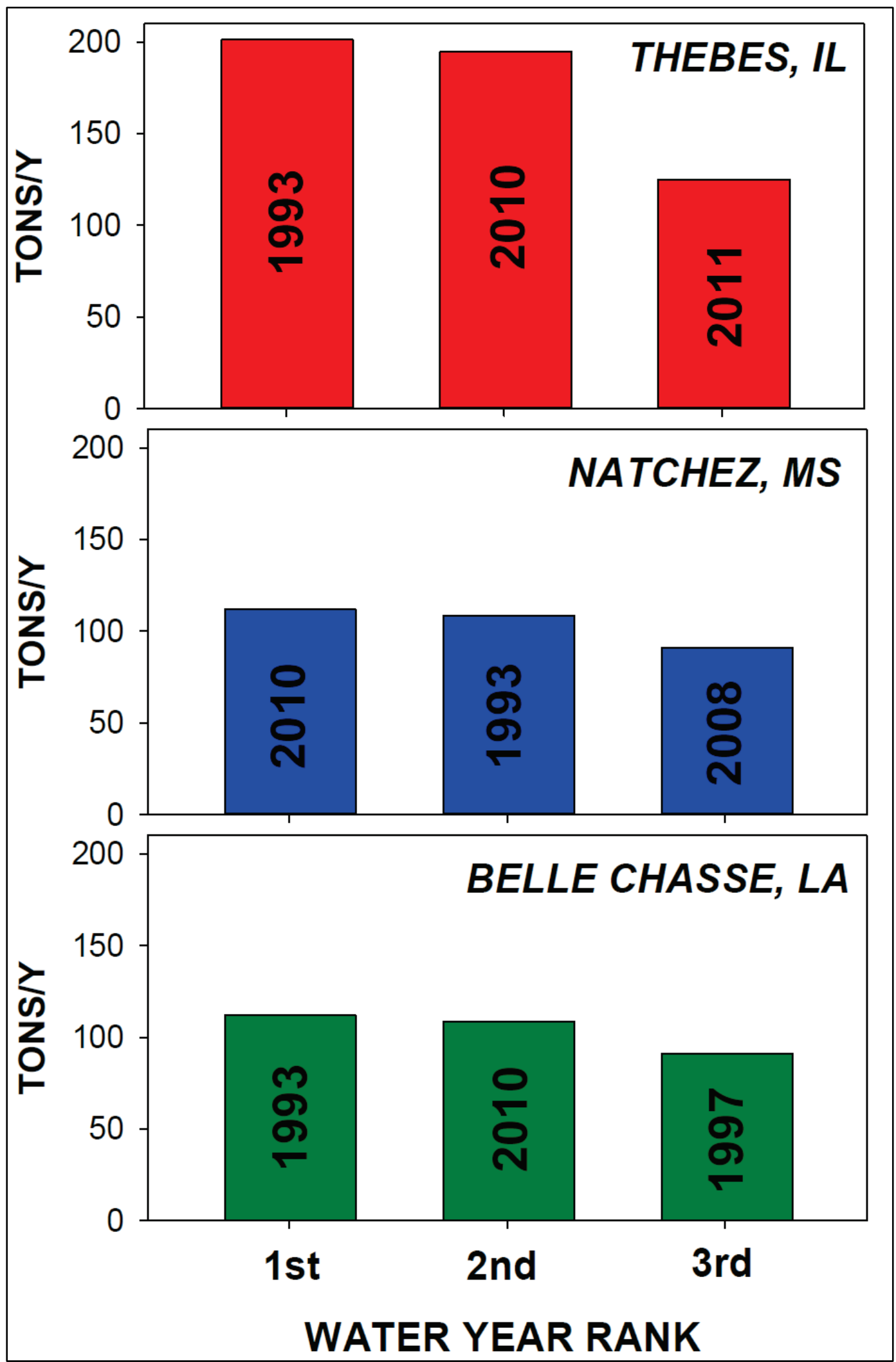


Figure 12. Annual total suspended sediment discharge calculated for each year in the WY 19902013 period for Mississippi River stations at Thebes, IL; Natchez, MS; and Belle Chasse, LA.



Variability associated with the tributary inputs also affect the sediment budgets calculated in Tables 6 and 7. Tributary monitoring stations are subject to the same data density and paucity of high flow event issues as the Mississippi River stations, decreasing the reliability of the results. In addition, the location of the last monitoring station at a significant distance upstream of their confluence with the Mississippi River leads to the possibility of second-order streams and bank/channel processes impacting the water and sediment reaching the Mississippi River in the intervening tributary reach. 
Tributary inputs are summarized in Figures 13 and 14 from the standpoint of their relative contribution to the overall Mississippi River discharge, as is the mud-sand ratio of their input, defined as the relative quantity of the mud $(<62.5 \mu \mathrm{m})$ and sand $(>62.5 \mu \mathrm{m})$ fraction in the total load. The Missouri and the Ohio Rivers dominate sediment input to the study reach as a whole for the 17 tributaries analyzed (Figure 13). Total suspended load input from the Missouri River, measured from the most downstream monitoring station at Hermann, MO, is $35,614 \%$ (>200,000\% sand) of that calculated at the station immediately below Gavin's Point Dam (i.e., Yankton, SD). As mentioned in 4.4.1, water flux increased 368\% in that Missouri River reach, representing the additive flow of multiple secondorder tributaries into the Missouri River below the dammed reach. While the enormous increase in sediment load may be carried by these secondorder rivers, the disproportionate increase in sediment load relative to water suggest a closer examination of this reach to determine if other mechanisms are contributing sediment. This examination is beyond the reach of the present study.

The relative input of mud versus sand of the 17 tributaries is highly variable, ranging from 56:1 in the Illinois River to 1.2:1 in the Arkansas River. This variability in sand input relative to fines in the suspended load is likely a complex interaction between each tributary in (1) basin geology, (2) hydrologic cycle, (3) elevation gradient, and (4) degree and function of modification by dams, locks, and bank armoring. Grain size variability also impacts the relative order of importance of sediment input into the Mississippi River by the 17 tributaries (Figure 14): the Missouri River is responsible for $47 \%$ of the fines input, but $65 \%$ of the sand input, while the sand-poorer Ohio River input is $20 \%$ and $10 \%$, respectively. The offset is even more dramatic in some of the smaller tributary inputs; the mud-rich Illinois and Yazoo Rivers contribute sixteen and six times as much mud as sand, respectively.

Sand budgeting (Table 7, Figure 9) station issues are similar to total load: a major deficit in the low confidence stations at St. Louis, MO, and Chester, IL, and another deficit in the USACE Vicksburg District stations relative to the inputs from the Mississippi River (Thebes, IL) and from major tributaries like the Ohio, Arkansas, and White Rivers. The only distinct difference in the sand load from the total load calculations is the drop between Baton Rouge and Belle Chasse, LA. Since total loads remained relatively stable between the stations (Table 6), it implies an 
increase in fines coincident with the decrease in sand. Allison et al. (2012) also observed this decrease in sand load and attributed it to the effects of dredge disposal of sand at the river crossing immediately upriver of the USGS Baton Rouge station as artificially increasing suspended sand loads.

Figure 13. Bar chart of total annual average suspended load in WY 1990-2013 divided into mud and sand fraction for tributaries entering the Mississippi River between St. Louis, MO, and Old River Control.

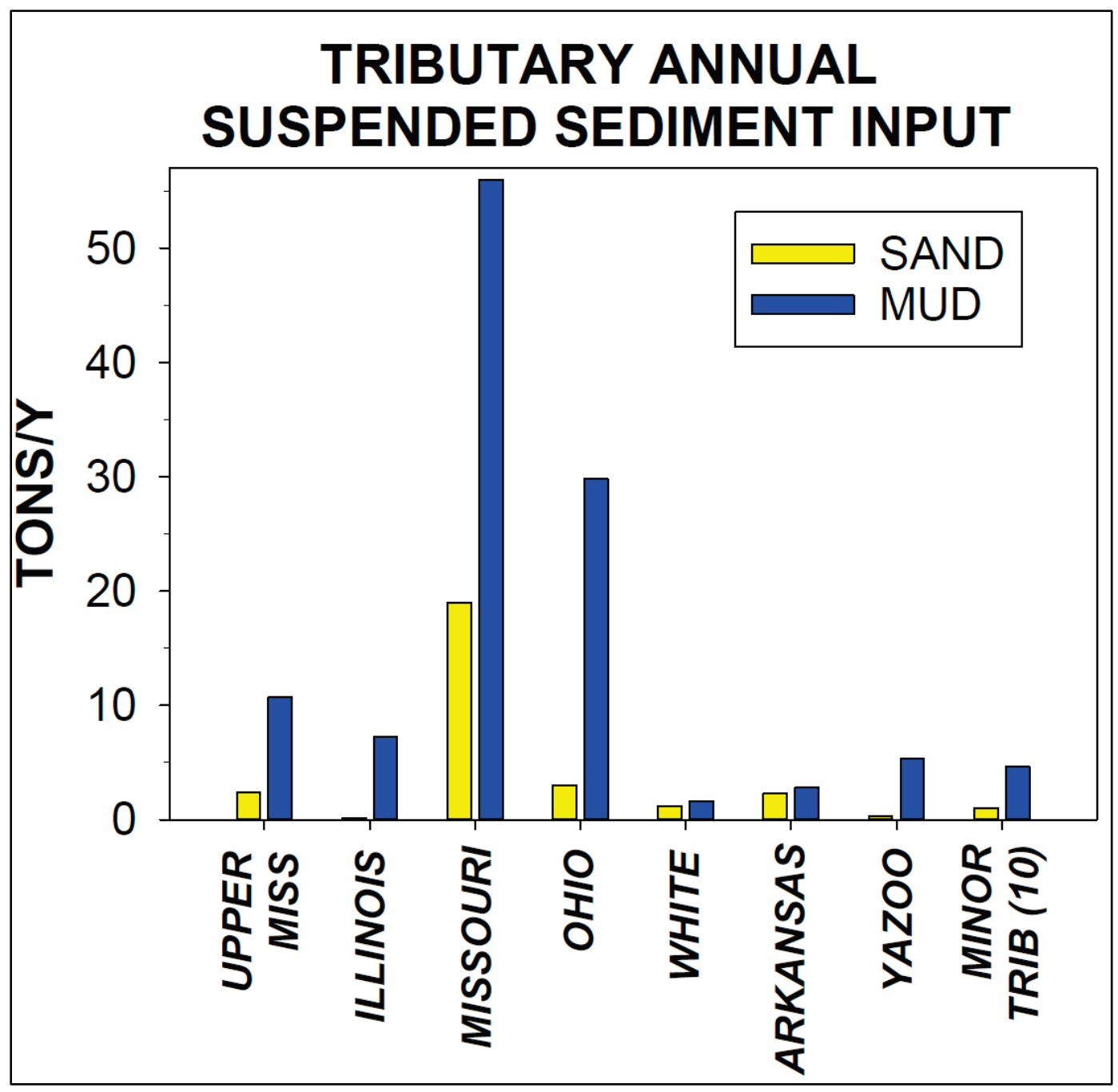


Figure 14. Pie chart of total annual average suspended load in WY 1990-2013 of tributaries divided into mud and sand fraction and plotted as percentage of the overall tributary sediment flux into the Mississippi River between St. Louis, MO, and Old River Control.

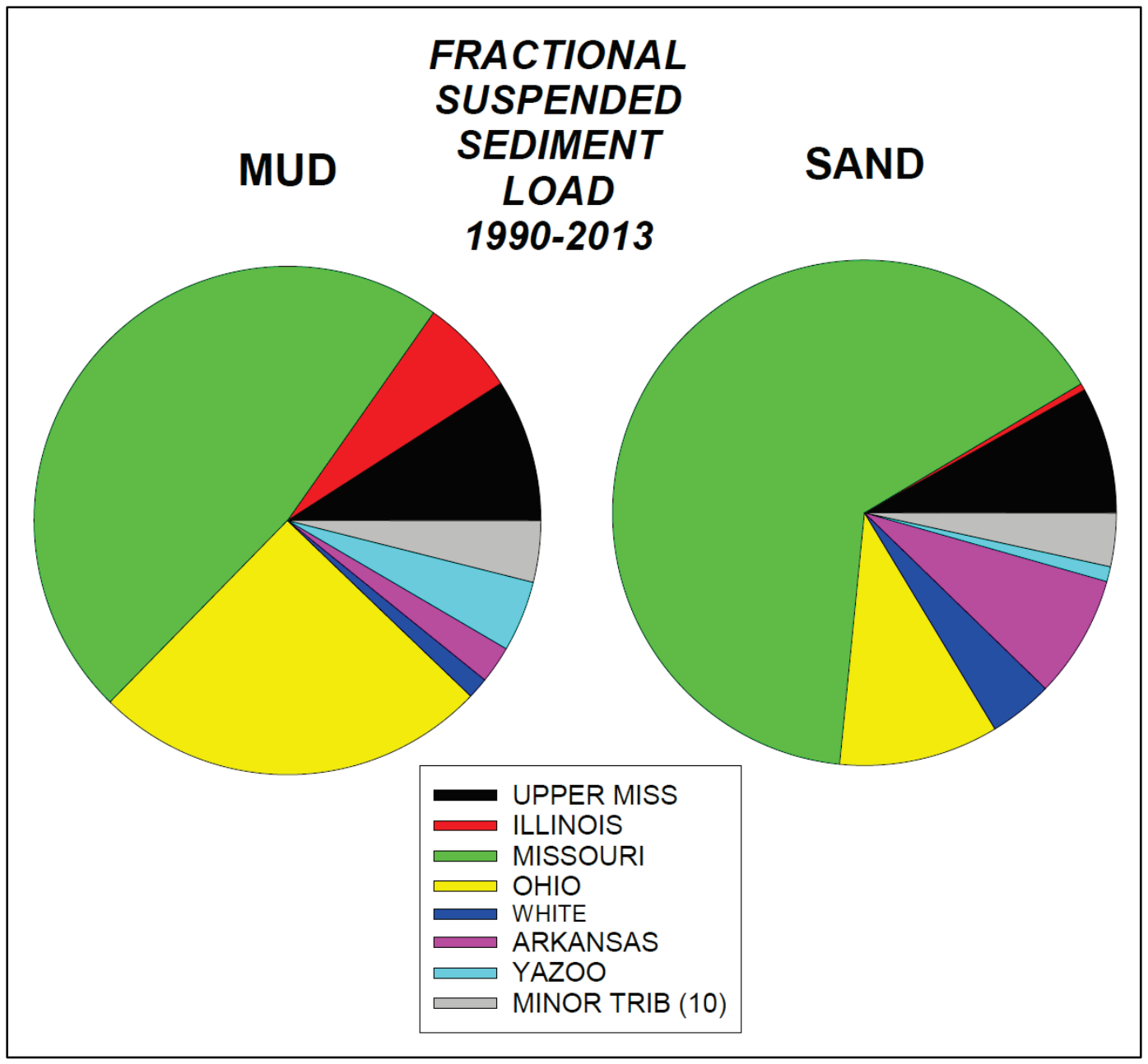

\subsection{A comparison of suspended sediment loads in WY 1990-2013 with WY 1970-1978}

As mentioned previously, the selection of tributaries and Mississippi River stations to examine in the present report was guided by the previous Keown et al. (1981) calculation of sediment budgets in the St. Louis, MO, to Gulf of Mexico reach. Hence, a major goal of the present report is to compare results with their earlier data. The Keown report calculates sediment budgets for two periods: WY 1970-1978 and pre-1953. Their purpose was to contrast a period prior to installation of major dams on the Missouri River and many other engineering modifications to the system, with a post-modification record. Most of the major modifications to the system have occurred prior to WY 1978, other than bank armoring of some sections and an increasing number of lock and dam structures on 
tributaries such as the Arkansas and Ohio Rivers. Minor modifications to the major tributaries are still taking place-the Olmstead Lock and Dam is scheduled to finish construction in 2020 and replace Lock and Dams 52 and 53 at a point on the Ohio River 17 miles upstream of the Mississippi River confluence. More significant modifications (e.g., dams) have taken place on smaller tributaries and their second-order streams since WY 1978. It is the impact of these activities on sediment budgets in the Mississippi River, as well as non-structural activities such as shifting land use and soil conservation practices, that can be examined by comparing the present WY 1990-2013 dataset with the Keown sediment budgets.

The Keown report did not quantify suspended sand load at Mississippi River or tributary stations, so the comparisons can only be made utilizing total suspended loads. Figure 15 shows a graphical station-by-station and tributaries most downstream station comparison between WY 1970-1978 and WY 1990-2013. The Missouri and Ohio Rivers are the first and second largest suspended sediment tributary inputs in both WY 1970-1978 and 1990-2013. All tributary inputs declined between the two time periods except the Yazoo River (5.6 vs. 4.2 million tons), but some declined more precipitously than others. Missouri River suspended sediment input (from Hermann, MO) fell only $13 \%$ over the interval while the Ohio River declined by $59 \%$ (Figure 16). Mississippi River stations, where directly comparable between studies (e.g., St. Louis, MO; Arkansas City, AR; Vicksburg and Natchez, MS), fell by an amount generally equivalent to the integrated tributary decrease in flux (227.4 to 147.4 million tons). Due to a smaller-than-average decline in suspended sediment load, the Missouri River became even more dominant in the total flux from the 17 tributaries contributing to the Mississippi River, from 38\% in WY $1970-1978$ to $51 \%$ in 1990-2013 (Figure 17).

The comparison with the pre-1953 suspended sediment loads calculated by Keown et al. (1981) is provided in Figure 18. Complications of direct comparison of this early data with the later compilations arise from (1) many tributaries lacked monitoring stations during this time period, (2) the number of Mississippi River stations was reduced during this time period, and (3) the absence of a controlled outflow at Old River Control makes relative contributions to the Atchafalaya River and Mississippi River distributaries difficult to quantify. However, several generalizations can be made. The magnitude of decline in sediment load induced by placements of the dams that virtually sealed off sediment coming from the 
Upper Missouri River (see Yankton, SD, loads, Figure 18) had a much larger impact on the Missouri River contribution than any effect post WY 1978. In contrast, the decline from the Upper Mississippi River has been more linear (e.g., 36.3 to 21 to 13.1 million tons). Much of the lock and dam and jetty confinement of the channel was conducted in the nineteenth and early twentieth century, prior to when even the pre-1953 data were collected. In the Arkansas River, there was a sharp decline between pre1953 and 1970-1978 (92.8 to 11.4 million tons). Major dam installation and bank stabilization on the Arkansas River occurred in the 1940s.

Figure 15. Annual average total suspended load (computed in $10^{6}$ tons/yr) for Mississippi River stations and Missouri River stations (red circles) and tributary inputs in the reach from St. Louis, MO, to Louisiana integrated for the periods of WY 1970-1978 (left; from Keown et al. 1981) and 1990-2013 (right). Asterisks in the Keown data (identified in that study) refer to small sample sets that make the calculated load only a first-order estimate.

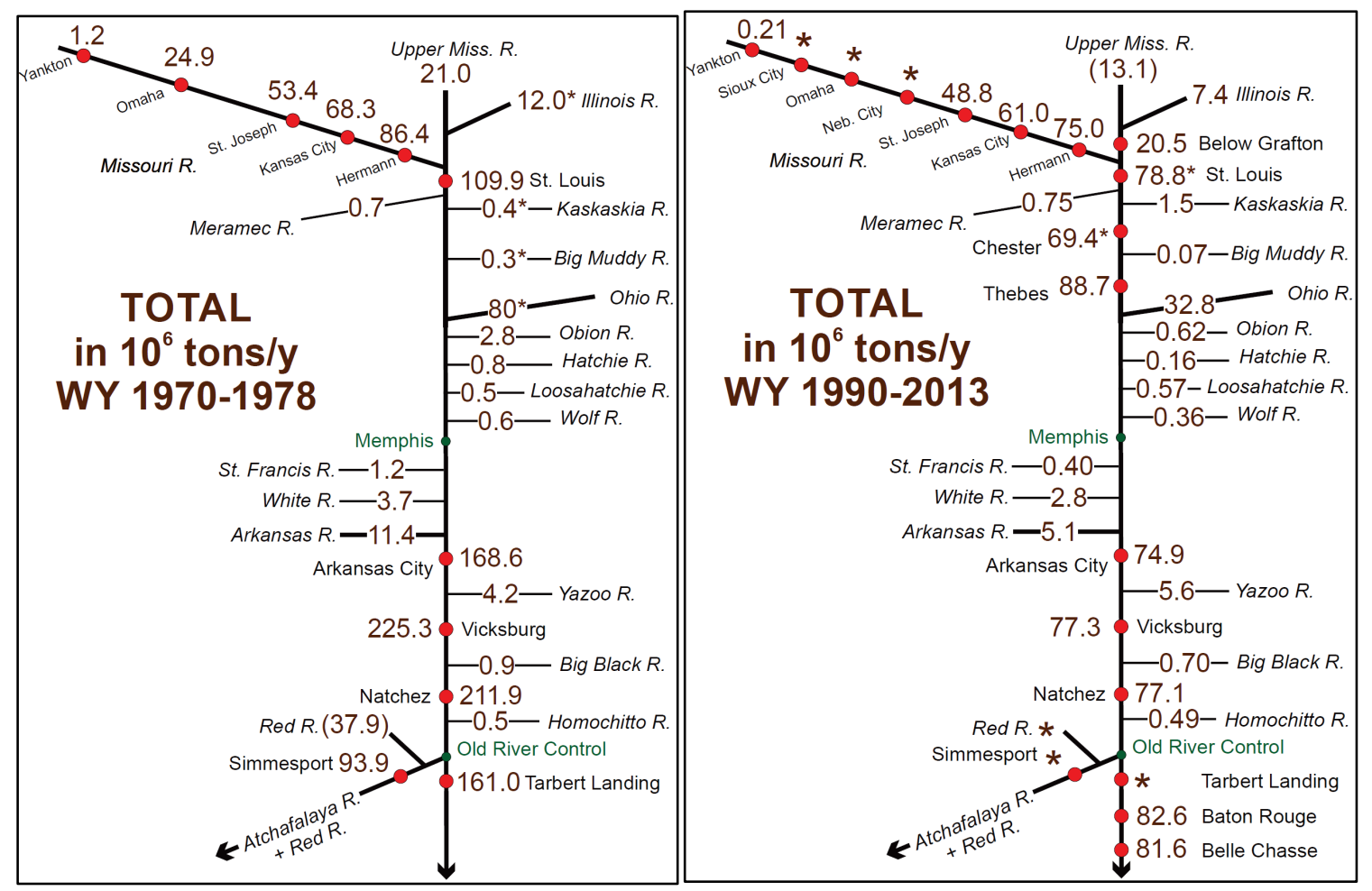


Figure 16. Bar chart of total annual average suspended load in WY 1970-1978 (from Keown et al. 1981) and WY 1990-2013 for tributaries entering the Mississippi River between St. Louis, MO, and Old River Control.

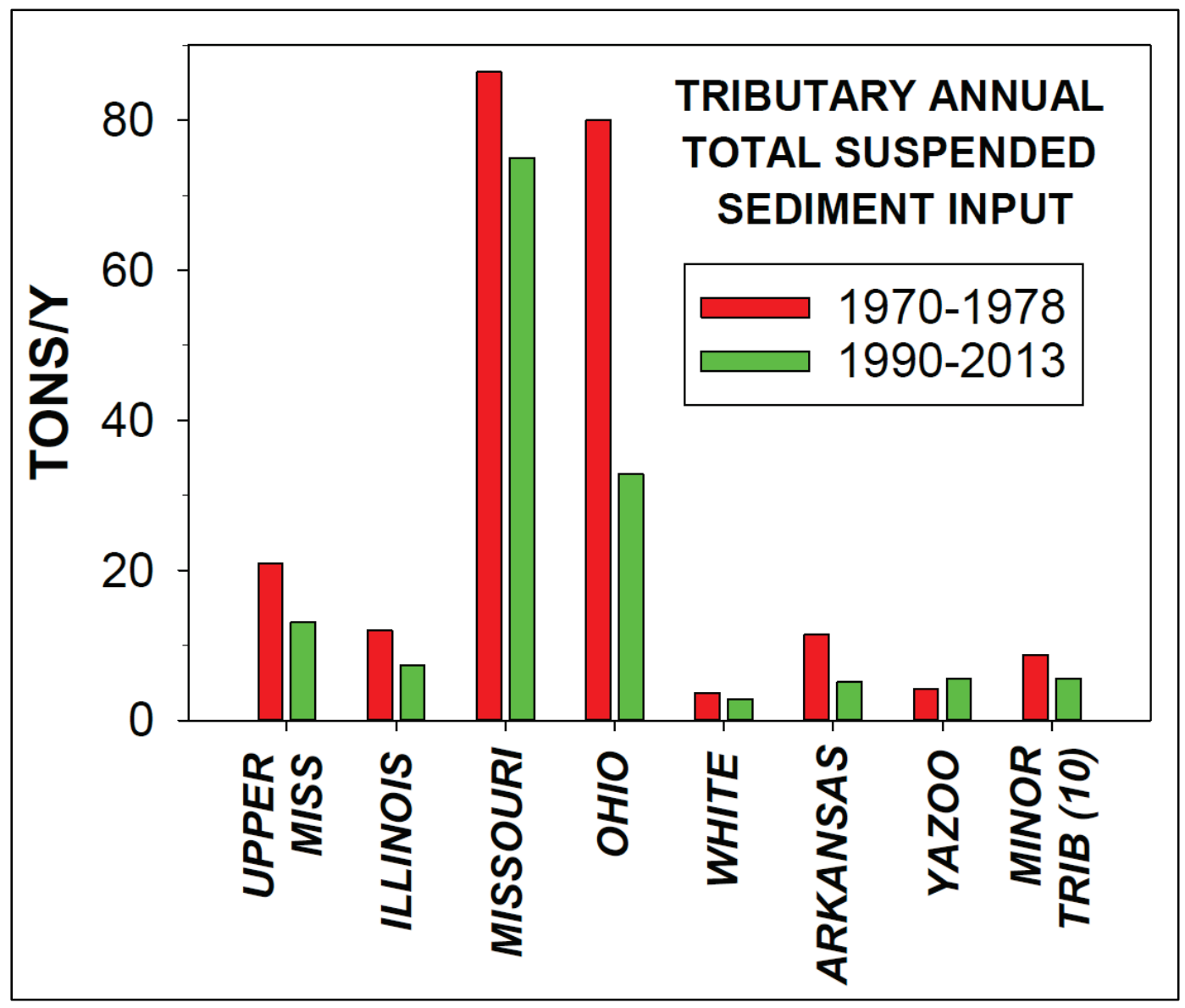


Figure 17. Pie chart of total annual average suspended load in WY 1970-1978 (from Keown et al. 1981) and WY 1990-2013 of overall tributary sediment flux into the Mississippi River between St. Louis, MO, and Old River Control.

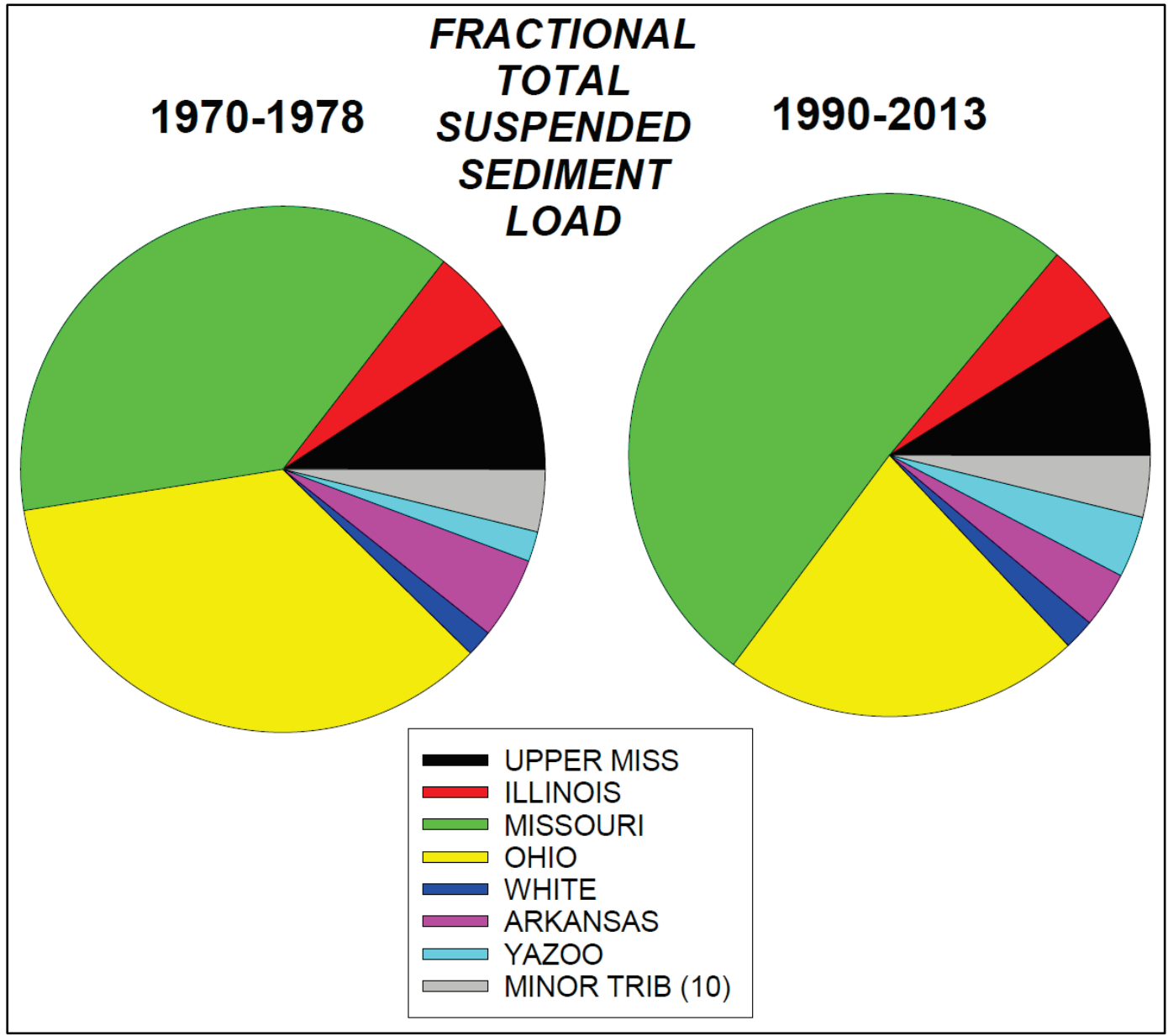


Figure 18. Annual average total suspended load (computed in $10^{6}$ tons/yr) for Mississippi River stations and Missouri River stations (red circles) and tributary inputs in the reach from St. Louis, MO, to Louisiana integrated for the periods of pre-1953 (left) and WY 1970-1978 (from Keown et al. 1981). Asterisks in the Keown data (identified in that study) refer to small sample sets that make the calculated load only a first-order estimate. Note that Old River

Control did not exist in pre-1953, and sediment flux going down the Atchafalaya River pathway was unregulated discharge from the Mississippi River plus flow from the Red River.


\subsection{Future network design and station operation}

The present location and sampling strategy employed in the Mississippi River and its tributaries is the result of more than $50 \mathrm{yr}$ of evolving network design and shifting priorities from the two Federal agencies tasked with this effort (i.e., USGS and USACE). There is a reluctance on the part of these agencies to discontinue any long-term monitoring sites because of their value in documenting historical changes to the Mississippi River's sediment load. This is true even when they are proven to have issues for suspended sediment monitoring. For instance, Allison et al. (2012) discuss issues with monitoring at St. Francisville, LA, a USGS site since the 1970s, which has been shown to have overbanking and flow (and sediment) loss when discharge exceeds approximately 700,000 ft $3 / \mathrm{sec}$. Even Tarbert Landing, MS, a key USACE site for triggering the operation of flood control structures in large floods, was documented as having overbank flow in the 2011 flood (Heitmuller et al. 2016). While it is not the place for the present study to suggest when stations should be closed or moved, because the authors' analytical methods did not isolate the effect 
of these complications, it is recommended that stations should be carefully examined by the operators to confirm that (1) measurements of water discharge and suspended sediment load throughout the discharge cycle are constrained within banks or that (2) the sampling regime is designed to sample overbank areas during periods where bank full discharge is exceeded at the site.

The water and sediment budgets outlined in previous sections rely on two main conceptual ideas with respect to examining morphological change as the Mississippi River flows from St. Louis, MO, to the Gulf of Mexico. The first is that regardless of the number of stations in the river itself, they should be spaced somewhat evenly apart. This allows for increases or decreases in the sediment load observed between stations to be linked to a relatively short river reach where morphodynamic causality can be examined. For example, if Station 2 is 50 miles downstream of Station 1, but Station 3 is 500 miles downstream, then the reach between Stations 1 and 2 is likely to be too short to show a measurable difference in suspended sediment load, even if the bed is aggrading or eroding. The large distance between Stations 2 and 3, while showing a measurable difference in the load if there are bed morphological changes, will be so long as to make pinpointing the zone of activity difficult. Spacing Stations 1-3 at 250 miles apart would balance the two factors. In future comprehensive reports where there is integratation of the sediment budget with morphological change and specific gage records, there is intent to make recommendations about what spacing would be ideal to see measurable sediment budget differences and link them to specific, observed changes occurring on the river's morphology.

The second conceptual idea for station location to examine morphological change in the Mississippi River is to site stations relative to tributary inputs. The increase in water, and hence stream power, associated with a large tributary can be anticipated to be a zone of morphological and sediment transport capacity change. Of the 17 tributaries analyzed by the present report and the Keown report, six major tributaries (i.e., Ohio, Upper Mississippi, Missouri, Arkansas, White, and Illinois Rivers) account for $93.4 \%$ of the tributary water input, and properly gaging their inputs and the changes they induce in Mississippi River suspended sediment budget is of the highest priority. 
While it is beyond the scope of this report to make recommendations for preferred station additions and alterations, some general observations follow, ordered from upstream to downstream (and not prioritized).

Upper Mississippi River Tributary - Suspended sediment load for the present report from the Upper Mississippi River is not measured directly but is subtracted from the total combined load at USGS Below Grafton (water discharge from USGS Grafton) of the Upper Mississippi and Illinois Rivers, and the measured flow of the Illinois River at USGS Valley City, IL (Table 3). In the Keown report, suspended sediment load was available from the USGS station at Hannibal, MO; however, boat-based sediment data from this station (USGS 05501660) was discontinued in 1984, although a stage record is available online beginning in 2011. Reactivation of USGS Hannibal, MO, would provide more accurate information for the Upper Mississippi River inputs (second-largest water input tributary) into the Mississippi River.

St. Louis, MO, and Chester, IL - While these stations are adequate to monitor the reach between the Missouri River and Thebes, IL, the limited dataset available to date (WY 2011-2014) has degraded quality of the sediment ratings curves for these stations. It is viewed as critical that the data collection activities initiated by the USGS in 2011 at these stations be continued to refine their sediment ratings curves. The high priority for continuance of the station at St. Louis, MO, stems from its location immediately below the confluence of the Missouri River, Upper Mississippi River, and Illinois River tributaries. However, given that the station at Thebes, IL, may be backwater affected by the Ohio River during certain conditions (see companion specific gage report ${ }^{1}$ ), the Chester, IL, station further upstream from Thebes, IL, can also be argued as valuable as well.

Thebes, IL, to Arkansas City, AR, Reach - While there are three USACE Mississippi River stations in the Vicksburg district, separated by approximately 194 river miles (120-Arkansas City to Vicksburg; 72Vicksburg to Natchez), the separation between Arkansas City, AR, and the next upstream station at Thebes, IL (USGS), is approximately 444 river miles. Eight tributaries enter the Mississippi River between Thebes, IL, and

\footnotetext{
1 Biedenharn, D. A., M. A. Allison, C. D. Little, Jr., C. R. Thorne, and C. C. Watson. In preparation. LargeScale Geomorphic Change in the Mississippi River from St. Louis, MO, to Donaldsonville, LA as Revealed by Specific Gage Records. MVD MRG\&P Technical Report. Vicksburg, MS: U.S. Army Corps of Engineers, Mississippi Valley Division.
} 
Arkansas City, AR, including the Ohio (first), Arkansas (fourth) and White (fifth) Rivers. An ideal station density would be an additional Mississippi River station immediately downstream of the Ohio River and a second immediately above the Arkansas River and below the White River to determine the impact of these two largest tributaries in this reach. If limited to a single station, a location between the White River and Ohio Rivers would be ideal. The USGS station at Memphis, TN (USGS 0732000), fits the single station locale possibility and has been collecting water discharge data since 1933 (USACE operates the stage gage at that locale). The station has also been occupied sporadically by the USGS for boat-based sediment load measurements in WY 1989-2011 (16 total suspended load measurements), but insufficient data exist to date to construct a sediment ratings curve. An alternative to upgrading data collection at the USGS station at Memphis, TN, would be for USACE Memphis district to initiate data collection at several stations along this critical reach.

Arkansas Tributary - Calculated inputs from the Arkansas River tributary were obtained from the station downstream of Terry Lock and Dam below Little Rock, AR (USGS 07263620). This station location differed from the Keown report, which utilized a station at Little Rock, AR, farther upstream. The sediment load data collection was presumably relocated due to the construction of several structures in the reach near Little Rock, AR. Even the present station utilized is approximately 116 river miles above the confluence with the Mississippi River. Relocation, or additional sediment load measurements closer to the Mississippi River confluence, would give a more accurate estimate of sediments escaping the highly modified (by lock and dam) reach. Adding sediment load measurements at the USGS station at Pendleton, AR (USGS 07265280), where stage is gaged, is one possibility.

In addition to station locations, another critical aspect of sediment budgeting revealed by the present study is the need to standardize field and laboratory methodology. Variability in these methods induces variation in station-by-station comparisons, and even in the time-series of a single station if methodologies are altered in the middle of the period of record. This standardization does not exist at present, in part because multiple federal agencies are involved (USGS, USACE) and methodologies differ even within USACE or USGS regional entity (e.g., district, water center). The two agencies are presently (2016) collaborating on resolving these issues. While an exact methodology to follow on the Mississippi 
River and its tributaries is beyond the present report and will need to be agreed upon by all the station operators involved, several general statements can be offered at this point. In terms of boat-based data collection, sampler type needs to be standardized, particularly with respect to weight, but standardizing type (e.g., depth-integrative, pointintegrative, weighted bottle) will also help minimize comparison inconsistencies. Some allowance can be made for when to switch sampler type/weight for lower flow velocities associated with low discharge periods in the Mississippi River or smaller tributary discharge. This sampler switch through the discharge cycle is already done at some USGS stations but is not standardized throughout basin. Given that virtually all the samplers utilized by the USGS and USACE were developed by their joint Federal Interagency FISP at Vicksburg, MS, they should be available and approved for use by all present operators on the river. Other field factors that induce inconsistencies include varying the number of verticals collected along the cross section and depth of each sample in the vertical.

Many stations in the Mississippi River network only analyze the suspended load sand fraction by conducting a sand-mud split. A more detailed sand-size breakdown (e.g., 62.5 to $125 \mu \mathrm{m}$-very fine sand; 125 to $250 \mu \mathrm{m}$-fine sand; 250 to $500 \mu \mathrm{m}$-medium sand; 500 to $1000 \mu \mathrm{m}$-coarse sand; and 1000 to $2000 \mu \mathrm{m}$-very coarse sand) of samples would allow for closer examination of the stream power associated changes in the grain size of the suspended load. This includes determining what size fractions constitute wash load versus bed material load in various reaches of the Mississippi River.

A variety of laboratory methods are also applied for determining weight of total sediment and sand fraction in sample bottles. While it is beyond this report to make recommendations for a preferred methodology, general observations can be made. Differences in (1) methodology for isolating the sediment fraction induced by oven-drying versus filtration, (2) sample splitting for aliquots to accomplish multiple analytical procedures and (3) analytical method (sieving, settling, laser diffraction, etc.) will induce variability in the resultant suspended sediment concentration and grain size breakdown for a sample.

Station operators are often loath to alter the station location, sampling strategy, or laboratory methodology at a long-term monitoring station to avoid a "step" in the sediment load data showing an artificial, 
methodologically induced increase or decrease in suspended sediment load. However, observations of the existing datasets where information is available indicate that virtually all stations that have operated over at least several decades have evolved in sampler type through the years, most have changed sampling strategy (e.g., number of verticals, number and depth of samples), and some have even changed locations over the period of record (e.g., Tarbert Landing, MS). If there are already steps in the data, whose impact is difficult to differentiate from real changes in Mississippi River and tributary suspended sediment load, then a basin-wide step, that is well-defined in time, can be viewed as less negative in impact. In addition, if this switch is accompanied by overlapping old-versus-new measurement data collection strategy for a period of time $(\sim 1-2 \mathrm{WY})$, not only will the impact on the sediment load step be quantifiable, but additional insight will be gained into the impact of historical changes in field and laboratory method on the calculated suspended load. 


\section{Summary}

Understanding historical and present-day fluvial processes and morphological responses in the Mississippi River is essential to designing and delivering long-term management of the system for flood control, navigation, and ecology that is cost effective, adaptable, and sustainable. Generation and initial assessment of suspended sediment ratings curves for the period of WY 1990-2013 for 12 Mississippi River and Atchafalaya River stations and 17 tributaries in the reach between St. Louis, MO, and the Gulf of Mexico has demonstrated that complex morphologic adjustments have occurred and are occurring throughout the river system that influence the sediment budget of the river. This study has also recognized that interpreting these effects is complicated by differences in field and laboratory methodology between stations, and in some stations, within the period of record that impacts the calculated total and sand-suspended load. Despite these complications, comparison with the Keown report of the WY 1970-1978 period indicates that the suspended sediment load of the Mississippi River and virtually all its tributaries continues to decrease decades after system alteration through dams, lock and dams, cutoffs, bank armoring, and land-use changes has slowed. Although no attempt was made in the present study to develop a causality linking the numerous natural and anthropogenic factors to observed sediment load changes observed in the river, the sediment ratings curves and suspended sediment loads (average and annual) developed in this study provide a powerful framework that when combined with other geomorphic assessment tools (e.g., specific gage records and channel morphological changes) will aid in unraveling the complex morphological process that drive the Mississippi River. 


\section{References}

Allison, M. A., C. R. Demas, B. A. Ebersole, B. A. Kleiss, C. D. Little, E. A. Meselhe, N. J. Powell, T. C. Pratt, and B. M. Vosburg. 2012. A water and sediment budget for the lower Mississippi-Atchafalaya River in flood years 2008-2010: implications for sediment discharge to the oceans and coastal restoration in Louisiana. Journal of Hydrology 432(3):84-97.

Allison, M. A., M. T. Ramirez, and E. A. Meselhe. 2014. Diversion of Mississippi River water and sediment to ameliorate coastal land loss in Louisiana, USA. Water Resources Management 28(12):4,113-4,126.

Beverage, J. P. 1987. Determining true depth of samplers suspended in deep, swift rivers. Minneapolis, MN: U.S. Federal Interagency Sedimentation Project Report GG, St. Anthony Falls Hydraulic Laboratory.

Edwards, T. K., and D. G. Glysson. 1999. Field methods for measurement of fluvial sediment. U.S. Geological Survey Techniques of Water-Resources Investigations, Book 3, Chapter C2.

Gray, J. R., and F. J. M. Simoes. 2008. Estimating sediment discharge, in sedimentation engineering processes, measurements, modeling, and practice, manual 110. Edited by M. Garcia, 1,067-1,088. Reston, VA: American Society of Civil Engineers.

Heidel, S. G. 1956. The progressive lag of sediment concentration with flood waves. Transactions of the American Geophysical Union 37(1):56-66.

Heitmuller, F. T., P. F. Hudson, and R. H. Kesel. 2016. Overbank sedimentation from the historic A.D. 2011 flood along the Lower Mississippi River, USA. Geology. doi: 10.1130/G38546.1.

Horowitz, A. J. 2003. An evaluation of sediment rating curves for estimating suspended sediment concentrations for subsequent flux calculations: Hydrological Processes 17(17):3,387-3,409.

Horowitz, A. J. 2010. A quarter century of declining suspended sediment fluxes in the Mississippi River and the effect of the 1993 flood. Hydrological Processes 24(1):13-34.

Keown, M. P., E. A. Dardeau, and E. M. Causey. 1981. Characterization of the suspendedsediment regime and bed-material gradation of the Mississippi River basin. U.S. Army Corps of Engineers Waterways Experiment Station Potamology Program Report 1, v. 1. Vicksburg, MS: U.S. Army Corps of Engineers, Waterways Experiment Station.

Koltun, G. F., J. R. Gray, and T. J. McElhone. 1994. User's manual for SEDCALC, a computer program for computation of suspended-sediment discharge. U.S. Geological Survey Open-File Report, 94-459. 
Little, C. D., Jr., and D. S. Biedenharn. 2014. Mississippi River hydrodynamic and delta management study (MRHDM)-geomorphic assessment. ERDC/CHL TR-14-5. Vicksburg, MS: Engineering Research Development Center.

Moore, N. R. 1972. Improvement of the Lower Mississippi River and tributaries, 19311972. Vicksburg, MS: Mississippi River Commission.

Mossa, J. 1996. Sediment dynamics in the lowermost Mississippi River. Engineering Geology 45(1-4):457-479.

Mueller, D. S., and C. R. Wagner. 2009. Measuring discharge with acoustic Doppler current profilers from a moving boat. U.S. Geological Survey Techniques and Methods, 3A-22. http://pubs.water.usgs.gov/tm3a22.

Porterfield, G. 1972. Computation of fluvial-sediment discharge. U.S. Geological Survey Techniques of Water-Resources Investigations, Book 3, Chapter C3.

Ramirez, M. T., and M. A. 2013. Suspension of bed-material sand over lateral bars in the lower Mississippi River, southeastern Louisiana. Journal of Geophysical Research-Earth Surface Processes 118(2):1-20.

Rasmussen, P. P., J. R. Gray, G. D. Glysson, and A. C. Ziegler. 2009. Guidelines and procedures for computing time-series suspended sediment concentrations and loads from in-stream turbidity-sensor and streamflow data. U.S. Geological Survey Techniques and Methods, Book 3, Chapter C4.

Runkel, R. L., C. G. Crawford, and T. A. Cohn. 2004. Load Estimator (LOADEST)-A FORTRAN program for estimating constituent loads in streams and rivers. U.S. Geological Survey Techniques and Methods, Book 4, Chapter A5.

Szalona, J. J. 1982. Development of a bag-type suspended-sediment sampler. Minneapolis, MN, St. Anthony Falls Hydraulics Laboratory, Federal Inter-Agency Sedimentation Project, Report Y.

Van Sickle, J., and R. L. Beschta. 1983. Supply-based models of suspended sediment transport in systems. Water Resources Research 19(3):768-778.

Walling, D. E. 1977. Assessing the accuracy of suspended sediment rating curves for a small basin. Water Resources Research 13(3):531-538. 


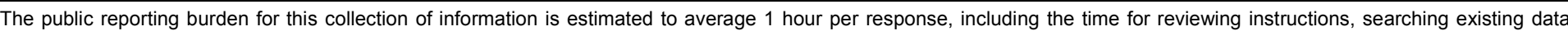

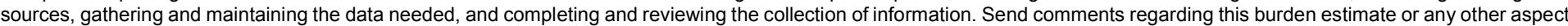

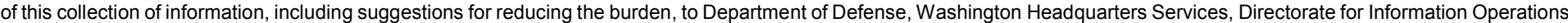

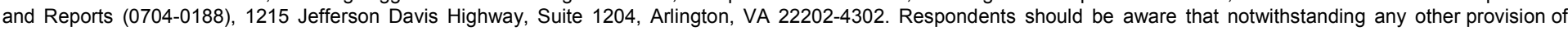
law, no person shall be subject to any penalty for failing to comply with a collection of information if it does not display a currently valid OMB control number. PLEASE DO NOT RETURN YOUR FORM TO THE ABOVE ADDRESS.
1. REPORT DATE
2. REPORT TYPE
July 2017
Final Report
3. DATES COVERED (From - To)

\section{TITLE AND SUBTITLE}

Suspended Sediment Loads and Tributary Inputs in the Mississippi River below St.

Louis, MO, 1990-2013: A Comparison with the Keown et al. (1981) Report

5a. CONTRACT NUMBER

5b. GRANT NUMBER

5c. PROGRAM ELEMENT NUMBER

6. AUTHOR(S)

Mead A. Allison, David S. Biedenharn, and Charles D. Little, Jr.

\section{5d. PROJECT NUMBER}

127672

5e. TASK NUMBER

5f. WORK UNIT NUMBER

8. PERFORMING ORGANIZATION REPORT NUMBER

MRG\&P Report No. 12

10. SPONSOR/MONITOR'S ACRONYM(S)

CEMVD MRG\&P

U.S. Army Corps of Engineers, Mississippi Valley Division

Mississippi River Geomorphology and Potamology Program

400 Walnut Street

Vicksburg, MS 39180

\section{SPONSOR/MONITOR'S REPORT} NUMBER(S)

\section{DISTRIBUTION/AVAILABILITY STATEMENT}

Approved for public release; distribution is unlimited.

\section{SUPPLEMENTARY NOTES}

\section{ABSTRACT}

Annual suspended sediment loads and water discharges were calculated in the Mississippi River main-stem channel and major tributary inputs at gaging stations from St. Louis, MO, to Belle Chasse, LA, for the period of water years 1990 to 2013 . The purpose was to (1) quantify changes in the Mississippi River sediment budget by linking with an earlier study for the periods of pre-1953 and 1970-1978 (Keown et al. 1981) and (2) examine the role of anthropogenic (e.g., dams, river control works, soil conservation practices) and natural (e.g., rainfall and denudation rates) factors in controlling these changes. The present report focuses on (1) a first-order comparison of changes in station-specific sediment loads with the earlier Keown results, (2) documenting data quality and data comparison issues observed in the data, and (3) identifying possible additional gaging station sites at critical junctures that would improve future sediment budget assessments for the Mississippi River. It is anticipated that sediment budgets will provide a powerful framework when combined with other geomorphic assessment tools (e.g., specific gage records, channel geometry changes) to aid in unraveling the complex morphological processes that drive the Mississippi River. This integration is also planned for future, comprehensive MRG\&P efforts.

\section{SUBJECT TERMS}

Flood control-Environmental aspects, Hydrology, Mississippi River Watershed, Sediment transport, Stream measurements, Suspended sediments

16. SECURITY CLASSIFICATION OF:

\begin{tabular}{|l|l|l|l|}
\hline a. REPORT & b. ABSTRACT & c. THIS PAGE & ABSTRACT \\
Unclassified & Unclassified & Unclassified & SAR
\end{tabular}

18. NUMBER OF 19a. NAME OF RESPONSIBLE PERSON PAGES

62
David S. Biedenharn 19b. TELEPHONE NUMBER (Include area code) $601-634-2923$ 
7. PERFORMING ORGANIZATION NAME(S) AND ADDRESS(ES) (continued)

The Water Institute of the Gulf

301 N. Main Street, Suite 2000

Baton Rouge, LA 70825

U.S. Army Engineer Research and Development Center Coastal and Hydraulics Laboratory

3909 Halls Ferry Road

Vicksburg, MS 39180-6199

Mendrop Resources Engineering, LLC

854 Wilson Drive, Suite A

Ridgeland, MS 39157 